\title{
Constitution d'un paysage urbain et diffusion du modèle de la cité grecque en Carie à l'époque hellénistique
}

Constitution of a Cityscape and Diffusion of the Greek City-State Model in Caria in the Hellenistic Period

\section{Gabrièle Larguinat-Turbatte}

\section{OpenEdition}

Journals

Édition électronique

URL : https://journals.openedition.org/gaia/1376

DOI : 10.4000/gaia.1376

ISSN : 2275-4776

Éditeur

UGA Éditions/Université Grenoble Alpes

Édition imprimée

ISBN : 978-2-37747-199-7

ISSN : $1287-3349$

\section{Référence électronique}

Gabrièle Larguinat-Turbatte, «Constitution d'un paysage urbain et diffusion du modèle de la cité grecque en Carie à l'époque hellénistique », Gaia [En ligne], 22-23 | 2020, mis en ligne le 30 juin 2020, consulté le 09 décembre 2021. URL : http://journals.openedition.org/gaia/1376 ; DOI : https://doi.org/ 10.4000/gaia.1376

Ce document a été généré automatiquement le 9 décembre 2021.

Gaia. Revue interdisciplinaire sur la Grèce archaïque 


\section{Constitution d'un paysage urbain et diffusion du modèle de la cité grecque en Carie à l'époque hellénistique}

Constitution of a Cityscape and Diffusion of the Greek City-State Model in Caria in the Hellenistic Period

Gabrièle Larguinat-Turbatte

\section{Introduction}

La Carie hellénistique comprend de nombreuses villes : cités grecques côtières, villes cariennes au développement architectural parfois ancien et fondations royales macédoniennes (fig. $1^{1}$ ). 
Fig. 1. - La Carie à l'époque hellénistique.

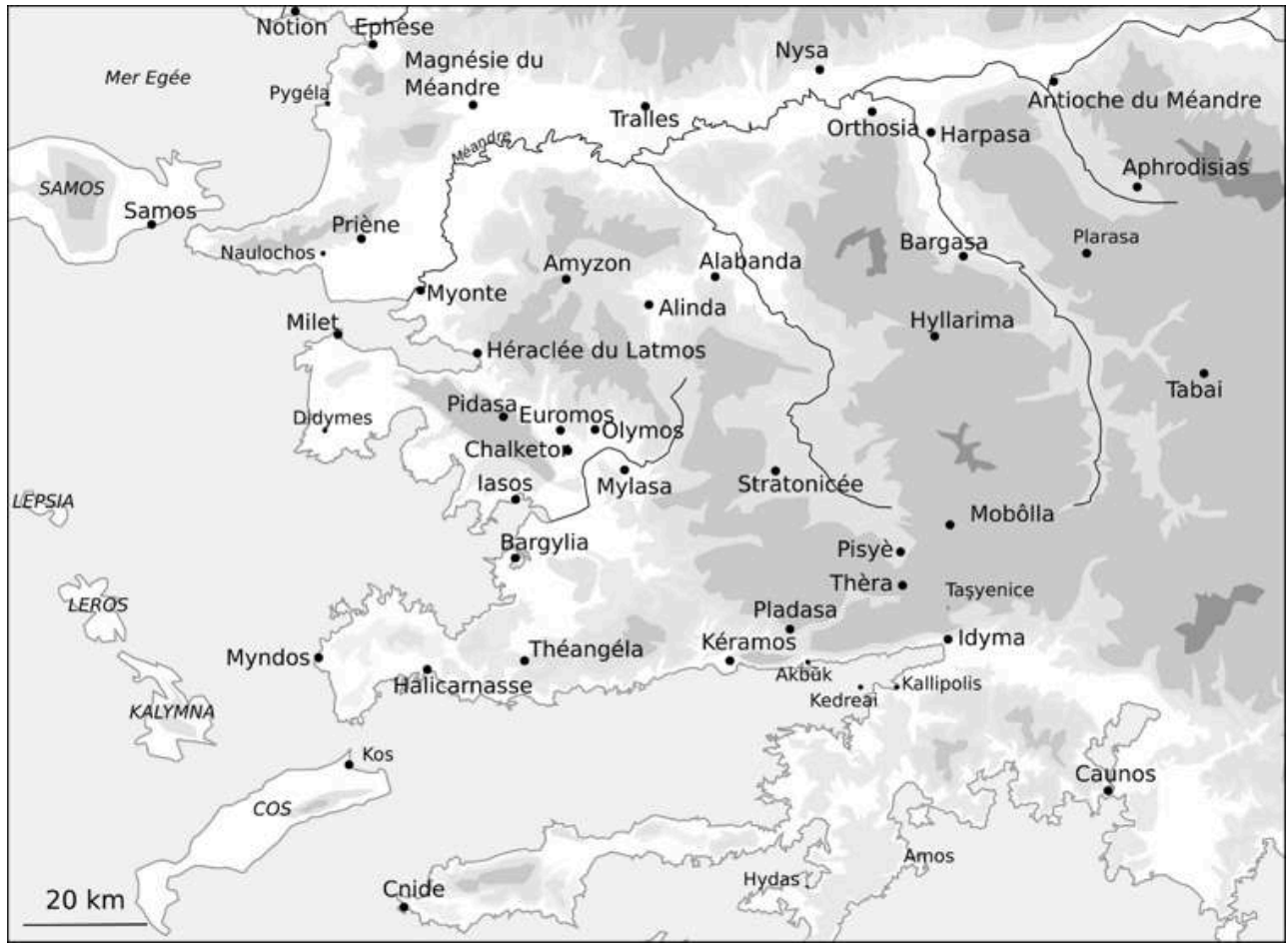

2 Les spécificités culturelles et géographiques de cette région ont été bien mises en évidence depuis les travaux de Jeanne et Louis Robert. Leur compréhension s'est affinée grâce aux prospections des équipes françaises et allemandes et aux progrès de la connaissance de la langue carienne et de l'onomastique ${ }^{2}$. Deux phénomènes majeurs sont à l'œuvre au cours de l'époque hellénistique. L'un est institutionnel, l'autre urbanistique. Amorcée au IV siècle $^{3}$ et parfois très avancée durant ce siècle dans les cités côtières, la diffusion du modèle de la cité grecque se généralise à l'ensemble de la Carie aux siècles suivants. Du point de vue de l'urbanisme, la constitution d'un paysage urbain de type grec paraît suivre le même cheminement. Plus précoce sur le littoral, il caractérise de nombreux sites de Carie intérieure où les cités, tout en conservant leur topographie traditionnelle, adoptent peu à peu une architecture grecque associée à des transformations urbanistiques destinées à régulariser la trame urbaine ${ }^{4}$. Il semble ainsi que dans l'intérieur de la Carie, où l'évolution est un peu plus tardive que sur la côte, la constitution d'un paysage urbain accompagne le processus de poliadisation, c'est-à-dire la transformation de ces communautés en cités de modèle grec ${ }^{5}$.

Les changements politiques et urbains en question relèvent du phénomène de $l^{\prime}$ 'hellénisation ${ }^{6}$, une notion opérante ici puisqu'il s'agit bien de l'adoption des formes architecturales typiquement grecques que sont les édifices politiques, les théâtres et les gymnases, associée à celle de la forme politique de la cité grecque. Pour autant, hellénisation ne suppose pas déculturation, puisque les communautés hellénisées de Carie conservent certains traits spécifiques à leur identité carienne ${ }^{7}$. Voulue par les Hécatomnides dès le $\mathrm{IV}^{\mathrm{e}}$ siècle ${ }^{8}$, elle s'accentue progressivement aux siècles suivants ${ }^{9}$. Pierre Debord a montré que le gradient de l'hellénisation diminue depuis le littoral vers l'intérieur des terres, notamment du fait de son ancienneté dans les régions de domination hécatomnide. Par comparaison avec l'Ionie voisine ou avec les cités côtières cariennes, l'hellénisation est plus tardive et moins profonde en Carie intérieure. C'est 
au cours de l'époque hellénistique qu'elle devient plus visible et plus prégnante dans tous les domaines, le $\mathrm{II}^{\mathrm{e}}$ siècle constituant un moment d'accélération des transformations ${ }^{10}$.

L'attention sera portée ici à certains bâtiments caractéristiques de la vie civique grecque: les édifices politiques, les théâtres et les gymnases. Pour l'époque hellénistique, il n'est pas question de reprendre les mots de Pausanias au sujet de Phanopeus en Phocide, qui transcrit les représentations des Grecs du II ${ }^{\mathrm{e}}$ siècle apr. J.-C., pour lesquels une ville sans bureau des magistrats (archeia), sans gymnase, sans théâtre et sans agora n'est pas une cité ${ }^{11}$. Pour l'époque hellénistique où les paysages urbains sont en construction, un tel jugement ne peut être systématisé même si c'est vers ce modèle que convergent peu à peu les villes de Carie.

5 Les vestiges archéologiques et les inscriptions s'éclairent mutuellement pour comprendre les transformations institutionnelles, culturelles et urbaines de ces sociétés ${ }^{12}$. En dressant la liste des édifices attestés par l'ensemble des sources, et en portant l'attention sur ceux dont la date de construction ou de réfection peut être appréhendée, il est possible de percevoir les rythmes d'avancée de la fabrique urbaine, c'est-à-dire du processus de construction de la ville perçu comme le reflet des choix des acteurs et de la situation des communautés ${ }^{13}$. L'ensemble de la documentation révèle des pratiques caractéristiques des cités du reste du monde grec associées à un certain nombre de spécificités cariennes.

6 À partir de l'époque hellénistique, les communautés ayant adopté un fonctionnement institutionnel grec se dotent d'édifices spécifiques propices au bon déroulement de la vie politique. De même, la construction de théâtres et de gymnases mobilise les ressources des cités au fur et à mesure que se diffusent les pratiques culturelles grecques $^{14}$. Le rythme de la transformation des espaces urbains est régulier, avec une accélération au cours du $\mathrm{II}^{\mathrm{e}}$ siècle. Enfin, la documentation épigraphique montre que la prise de décision se fait conformément aux pratiques grecques, de même que le financement qui fait appel à tout l'éventail des possibilités dont disposent les cités. Cette diversité des ressources financières a facilité la constitution d'un paysage urbain de type grec.

\section{La vie politique}

7 L'ensemble des édifices politiques destinés à abriter les institutions civiques est attesté en Carie à l'époque hellénistique (tableau 1$)^{15}$. 
Tableau 1. - Tableau des édifices politiques attestés en Carie.

\begin{tabular}{|c|c|c|c|}
\hline Ville & Bouleutérion & Prytanée & Autres \\
\hline Alabanda & $\mathrm{A}: \mathrm{II}^{\mathrm{e}}$ siècle & & \\
\hline Bargylia & & $\begin{array}{l}\mathrm{E}: 270 / 261 \text { (IIasos 608) } \\
\mathrm{E}: c a 129-127 \text { (IIasos 612) }\end{array}$ & \\
\hline Caunos & $\begin{array}{l}\text { L : après } 88 \text { (App. } \\
\text { Mith., 23) }\end{array}$ & $\begin{array}{l}\text { E : } \text { II }^{\circ} \text { siècle (Marek, 2006, } \\
\left.n^{\circ} 19\right)\end{array}$ & \\
\hline Cnide & & & $\begin{array}{l}\text { Dicasterion, damosion: } \\
\mathrm{E}: \text { ca } 300 \text { (IKnidos 221) }\end{array}$ \\
\hline Halicarnasse & $\begin{array}{l}\mathrm{E}: \mathrm{III}^{\mathrm{e}} \text { siècle } \\
(\mathrm{BdI} 78 ; \text { OGIS 46) }\end{array}$ & E : 303-ca 290 (Hicks, 1881) & $\begin{array}{l}\text { Dicasterion: } \\
\left.\mathrm{E}: \mathrm{II}^{\mathrm{e}} \text { siècle (OGIS } 46\right)\end{array}$ \\
\hline Héraclée du Latmos & $\mathrm{A}: \mathrm{II}^{\mathrm{e}}$ siècle & & \\
\hline Iasos & $\begin{array}{l}\mathrm{E}: \mathrm{II}^{\mathrm{e}} \text { siècle } \\
(\text { IIasos 252) }\end{array}$ & $\begin{array}{l}\mathrm{E}: 1^{\mathrm{re}} \text { moitié } \mathrm{II}^{\mathrm{e}} \text { siècle } \\
(\text { II asos } 82)\end{array}$ & $\begin{array}{l}\text { Archeion: } \\
\text { E : ca } 333-323 \\
\text { (IIasos } 30 ; \text { IIasos 252) } \\
\text { Archeion prostatikon: } \\
\text { E : fin } \mathrm{II}^{\mathrm{e}} \text {-début } \mathrm{II}^{\mathrm{e}} \mathrm{s} \text {. } \\
\text { (Maddoli, 2001) } \\
\text { E : début } \mathrm{II}^{\mathrm{e}} \text { siècle } \\
\text { (Maddoli, 2007) } \\
\text { Agoranomion: } \\
\text { A, E : } \mathrm{II}^{\mathrm{c}} \text { siècle (IIasos 22) }\end{array}$ \\
\hline Nysa & & & $\begin{array}{l}\text { Gérontikon: } \\
\mathrm{L}: \text { fin de l'époque } \\
\text { hellénistique (Str. 14.1.43) }\end{array}$ \\
\hline Stratonicée de Carie & $\begin{array}{l}\text { A : tardo- } \\
\text { hellénistique? }\end{array}$ & & $\begin{array}{l}\text { Stratègion: } \\
\text { E : milieu III siècle } \\
\text { (IStratonikeia } 1505)\end{array}$ \\
\hline Tralles & & $\mathrm{E}: \mathrm{III}^{\mathrm{e}}$ siècle (ITralleis 25 ) & $\begin{array}{l}\text { Ekklésiastèrion: } \\
\mathrm{L}: 1^{\mathrm{re}} \text { moitié du } \mathrm{I}^{\mathrm{er}} \text { siècle? } \\
\text { (Vitr. VII, } 5,5-7)\end{array}$ \\
\hline
\end{tabular}

Des bouleutéria ont été mis au jour à Alabanda, Héraclée du Latmos et Stratonicée de Carie (fig. 2$)^{16}$. Ils ne se démarquent pas de ceux d'autres régions du monde grec et présentent une architecture similaire à celle des bouleutéria d'Ionie dont ils pourraient être les contemporains ${ }^{17}$. Ainsi, au-delà de l'adoption d'un fonctionnement institutionnel grec, les cités cariennes choisissent les mêmes types architecturaux que ceux employés par leurs voisines ioniennes. D'autres bouleutéria sont mentionnés dans des inscriptions à Halicarnasse et à Iasos (III ${ }^{\mathrm{e}}$ et $\mathrm{II}^{\mathrm{e}}$ siècles) ${ }^{18}$. Enfin celui de Caunos est connu grâce à un épisode raconté par Appien en $88^{19}$.

Fig. 2. - Bouleutéria de Carie.

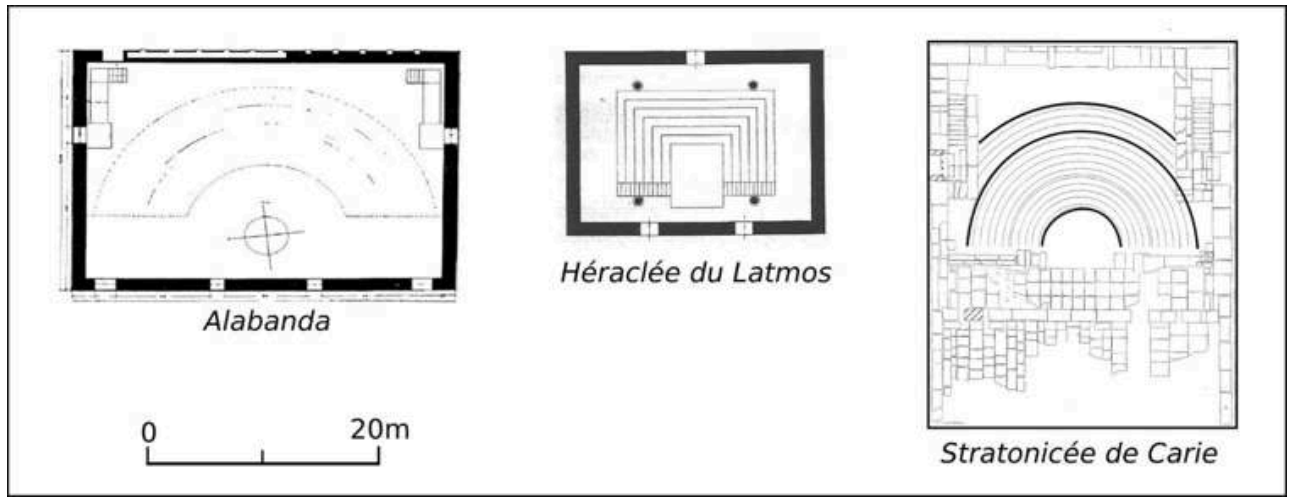

D’après Edhem Bey (1905, fig. 3), Krischen (1941, pl. 25), Mert (2008, fig. 131).

$9 \quad$ Les lieux de réunion d'autres types d'assemblées sont aussi connus pour les dernières années de l'époque hellénistique: Vitruve mentionne un ekklèsiastèrion à Tralles et Strabon cite le gérontikon de $\mathrm{Nysa}^{20}$. 
l'invitation au prytanée (invitation simple ou sitèsis) constitue un honneur répandu pour les évergètes des cités. À l'époque du satrape Asandros (321/320), trois épimélètes sont invités au prytanée de Pidasa ${ }^{21}$. Dans les années ca 270-261, un juge de Téos reçoit l'hospitalité au prytanée de Bargylia ${ }^{22}$. Dans les années 250 à Iasos, des juges iasiens intervenus à Calymna sont invités au prytanée ${ }^{23}$. Au cours du III $^{\mathrm{e}}$ siècle, la cité de Tralles confère à un certain Alexandros la nourriture au prytanée ${ }^{24}$. Plus tard, au II siècle, c'est un ambassadeur de Smyrne qui est invité au prytanée de Caunos ${ }^{25}$.

11 À Bargylia, cette fonction d'hospitalité du prytanée est énoncée dans un décret pour Téos et un juge téien et vient enrichir l'exposé des motifs du décret honorifique pour Poseidonios datant des années qui suivent la guerre d'Aristonicos (129-127). L'importance de l'action de Poseidonios en faveur de la restauration du prytanée est accentuée par le rôle essentiel du lieu dans la vie civique ${ }^{26}$ :

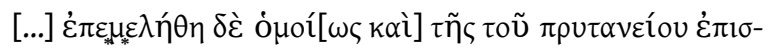

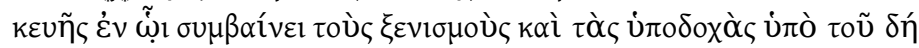

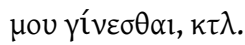

« [...] il a été chargé de la même manière de la restauration du prytanée dans lequel il arrive que des réceptions d'étrangers et des banquets publics soient organisés par le peuple $[. . .]^{27}$.»

Les tribunaux peuvent se réunir dans des édifices spécifiques : les dicasteria de Cnide et d'Halicarnasse sont clairement identifiés dans la ville (III siècle) ${ }^{28}$. Comme ailleurs dans le monde grec, les magistrats disposent de locaux nommés archeia : à Iasos, l'archeion est connu entre le dernier tiers $d u \mathrm{IV}^{\mathrm{e}}$ siècle et le $\mathrm{II}^{\mathrm{e}}$ siècle par des clauses de gravure, une dédicace et un décret honorifique ${ }^{29}$. Le local des agoranomes d'Iasos, l'agoranomion, a été identifié grâce à une inscription du II $^{\mathrm{e}}$ siècle gravée sur les piédroits de la porte de l'une des pièces construites à l'arrière de la stoa est de l'agora ${ }^{30}$. À Stratonicée de Carie, le local des stratèges, le stratègeion, sert de point de repère pour l'organisation de la défense de la ville (milieu du III ${ }^{\mathrm{e}}$ siècle) ${ }^{31}$.

Les premières attestations (archéologiques et épigraphiques) d'édifices politiques dans des cités cariennes datent de la fin $\mathrm{du}_{\mathrm{IV}}^{\mathrm{e}}$ siècle, comme dans la région voisine de l'Ionie. La Carie adopte les mêmes pratiques architecturales et épigraphiques et connaît une hellénisation et une poliadisation plus poussée dès les premières années de l'époque hellénistique ${ }^{32}$, y compris dans des communautés conservant d'importants traits cariens jusque dans les siècles suivants ${ }^{33}$.

\section{Les théâtres}

Les cités cariennes se dotent d'un théâtre de bonne heure, peut-être dès la fin de l'époque classique (Halicarnasse ${ }^{34}$ et Iasos $^{35}$ ), pour la plupart au cours de l'époque hellénistique (tableau 2) ${ }^{36}$. 
Tableau 2. - Théâtres de Carie.

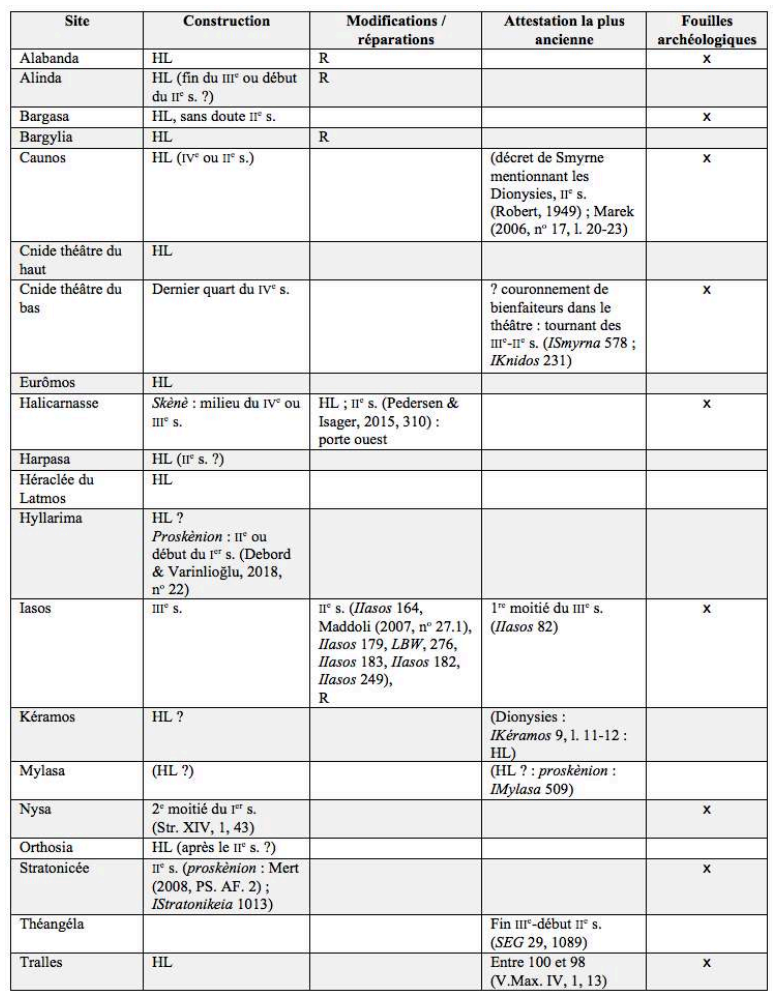

15 Généralement la chronologie est difficile à établir. Dans la plupart des cas, l'étude architecturale conduit à dater approximativement les édifices de l'époque hellénistique comme à Alinda (fig. 3), Bargasa et Eurômos ${ }^{37}$. Où qu'ils soient en Carie, les théâtres, souvent construits en matériau local, ne présentent pas de caractéristiques architecturales spécifiques.

Les inscriptions font état de travaux d'amélioration des édifices préexistants au II siècle où le théâtre d'Halicarnasse est orné d'une porte monumentale ${ }^{38}$ et où Hyllarima et Stratonicée de Carie dotent leur théâtre d'un proskènion de marbre ${ }^{39}$.

Fig. 3. - Théâtres d'Alinda (entrée nord-est), d'Héraclée du Latmos (escaliers et gradins) et de Cnide (koilon du théâtre du bas) (de gauche à droite).
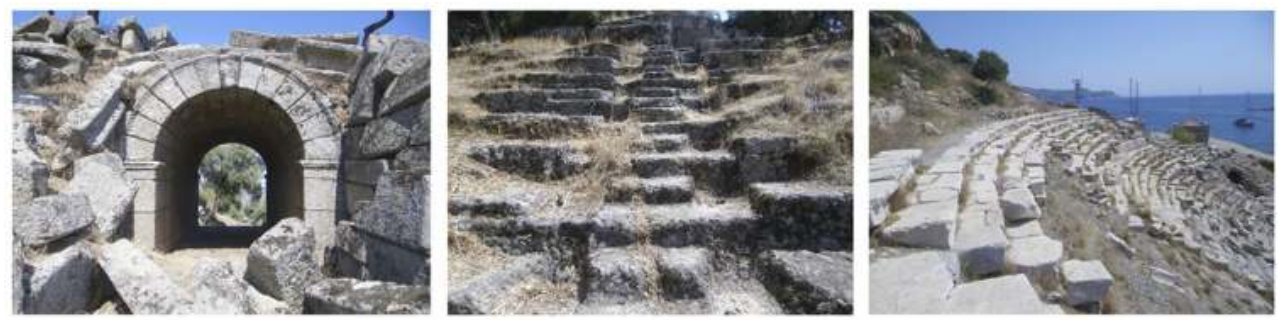

À Stratonicée de Carie, le théâtre occupe le versant nord d'une colline située au sud de la ville et devait s'inscrire dans la trame urbaine orthogonale. À l'exception du proskènion, l'édifice n'est pas daté avec précision. Louis Robert proposait une construction au $\mathrm{III}^{\mathrm{e}}$ siècle, à l'époque de la fondation de la ville $\mathrm{e}^{40}$, mais rien ne permet de le confirmer actuellement. Le koilon est particulièrement bien conservé dans sa partie inférieure, à l'exception de ses extrémités nord où les gradins se sont affaissés probablement suite à des mouvements de terrain. Le théâtre est adossé à la pente 
naturelle de la colline et présente un plan et des dimensions similaires à celles de nombreux autres théâtres micrasiatiques de l'époque hellénistique et du début de l'époque impériale $(c a 75 \times 80 \mathrm{~m})^{41}$. Son koilon en demi-cercle outrepassé est limité au nord par l'analemma construit dans un appareil caractéristique des édifices de la ville et dont les crampons métalliques ont fait l'objet d'une récupération systématique ${ }^{42}$. Seule la partie inférieure du koilon appartient à l'époque hellénistique, la volée de gradins supérieure datant probablement de l'époque impériale ${ }^{43}$. L'accès à l'orchestra se fait par deux portes de parodos dont celle située à l'est demeure en place. L'orchestra de 21,60 m de diamètre, avec son sol en terre battue, est bordée par un banc de proédrie en marbre blanc (fig. 4).

Fig. 4. - Stratonicée de Carie : le théâtre vu du nord.

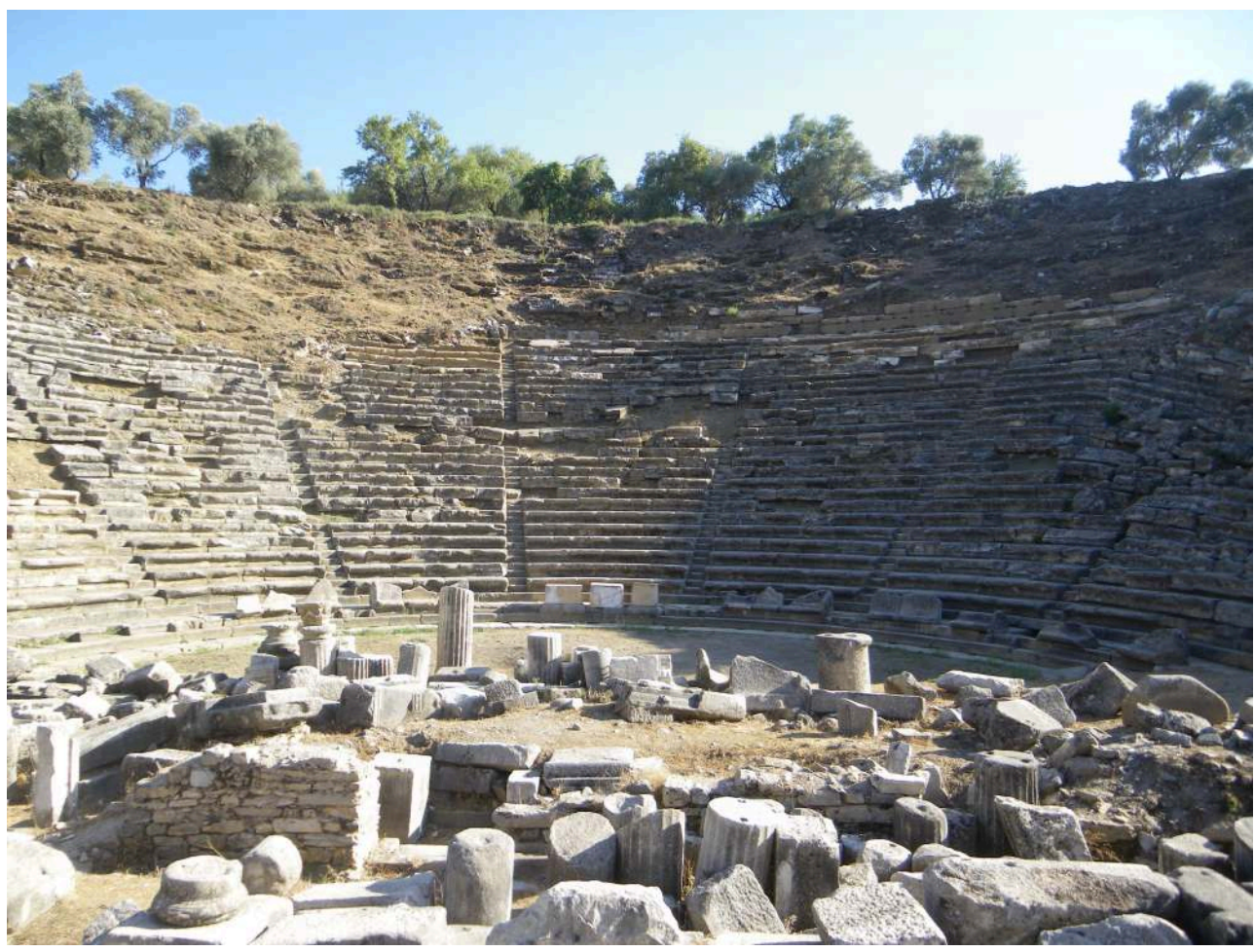

Seules les fondations de la skènè de $c a 5,20$ x $16,70 \mathrm{~m}$ sont conservées. Le proskènion financé par des évergètes locaux (ca 2,88 x 18,70 m) présente en façade 10 demicolonnes doriques cannelées placées entre deux piliers ${ }^{44}$. Certains entrecolonnements étaient pourvus de portes. Au-dessus des demi-colonnes, l'architrave dorique constituée de blocs d'architrave-frise porte les dédicaces de différents éléments du proskènion. Sa réalisation a dû s'étaler sur plusieurs décennies au II $^{\mathrm{e}}$ siècle, comme l'indiquent la paléographie des dédicaces et les différences de traitement des triglyphes de la frise ${ }^{45}$ (fig. 5). 
Fig. 5. - Stratonicée de Carie : bloc d'architrave-frise du proskènion portant la dédicace de Minniôn fils de Léôn et de ses fils.

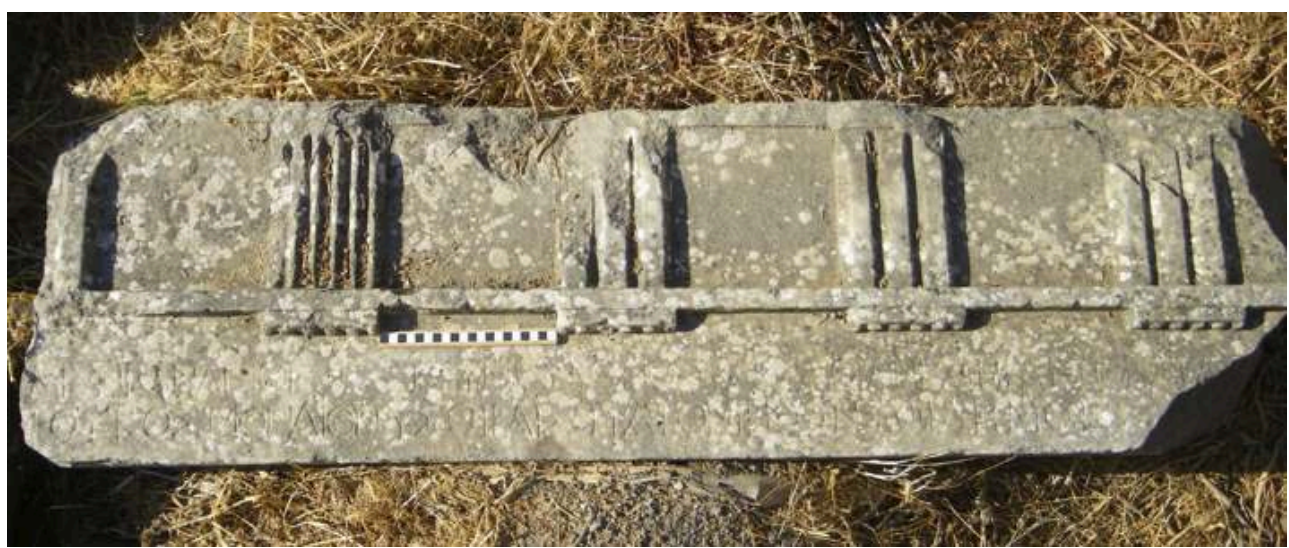

La dédicace suivante est gravée sur le bloc reproduit ci-dessus ${ }^{46}$ :

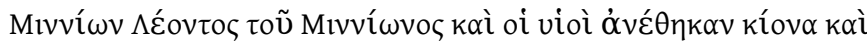

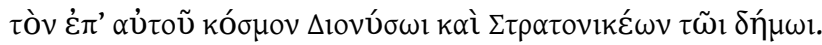

«Minniôn fils de Léôn fils de Minniôn et ses fils ont consacré la colonne et l'ornementation qui la surmonte à Dionysos et au peuple des Stratonicéens. »

20 Par ailleurs, et même si cela constitue une exception, il n'est pas nécessaire qu'un établissement soit une cité pour qu'il soit pourvu d'un théâtre. C'est le cas de Kastabos en Chersonèse de Carie, dont le sanctuaire d'Hémithéa constitue le lieu de réunion du koinon des Chersonésiotes. Ce koinon regroupe quinze communautés cariennes sous domination rhodienne depuis leur intégration à la Pérée (ca 300). Bien qu'ayant le statut de dèmes après cette date, les communautés des Chersonésiotes continuent de se réunir dans le sanctuaire d'Hémithéa, une déesse guérisseuse associée à une source. Ils érigent un théâtre au cours de l'époque hellénistique, dont le koilon délimité par un mur d'analemma est adossé à la pente naturelle du terrain et pourvu d'une skènè et d'un proskènion ${ }^{47}$. L'édifice est destiné à recevoir des représentations dramatiques et peut aussi servir de lieu de rassemblement du koinon ${ }^{48}$. Le cas de Kastabos et du koinon des Chersonésiotes est révélateur de l'adaptation du théâtre, forme architecturale et pratique culturelle grecques, à une forme politique typiquement carienne, le koinon. Le processus d'acculturation n'implique nullement un renoncement aux spécificités locales, les Chersonésiotes réussissant à conserver leur organisation politique traditionnelle malgré la domination rhodienne, ainsi que leur culte, par ailleurs pratiqué dans un cadre architectural grec ${ }^{49}$.

21 À côté des édifices formellement identifiés, les attestations de Dionysies ou d'autres fêtes célébrées dans des cités de Carie ne doivent pas conduire à restituer un théâtre dans chacune d'elles ${ }^{50}$. En effet, les représentations théatrales peuvent avoir lieu en dehors de tout édifice ${ }^{51}$. Par ailleurs, les archéologues ne parviennent toujours pas à localiser le théâtre, comme à Mylasa, où son emplacement fait débat ${ }^{52}$. Dans certains cas, les représentations pouvaient prendre place dans un théâtre construit en matériaux périssables n'ayant pas laissé de trace ${ }^{53}$.

Il est en tout cas très probable que la plupart des cités cariennes possèdent un théâtre, tant cet édifice est lié à la vie civique grecque. La présence de théâtres dans de petites cités de Carie intérieure comme Bargasa, Orthosia et la communauté vivant dans le site de Taşyenice montre que les populations étaient capables de mobiliser les ressources 
nécessaires quelle que soit leur taille ${ }^{54}$. Tous les types de villes peuvent être concernés, $\mathrm{y}$ compris celles de tradition non grecque où l'hellénisation est plus récente que dans les cités côtières ${ }^{55}$. Il est possible que dans les cités ayant adopté le modèle grec plus récemment, le théâtre perde une partie de sa signification politique et ne conserve que son rôle culturel et religieux, notamment parce que le public n'est pas nécessairement en mesure de saisir tout le sens politique des pièces qui sont représentées ${ }^{56}$. Néanmoins les références aux enjeux propres à un régime démocratique devaient faire sens dans des communautés se réclamant généralement de la démocratie ${ }^{57}$. Cet essor de la démocratie à l'époque hellénistique, avec toutes les nuances qui ont été mises en évidence par Philippe Gauthier notamment ${ }^{58}$, a vraisemblablement contribué au succès des concours dramatiques et à la diffusion du théâtre ${ }^{59}$. D'autre part, pénétrer à l'intérieur du théâtre et assister à des représentations dramatiques est d'ordinaire réservé aux citoyens et évergètes méritants ${ }^{60}$. Ainsi les émotions partagées à l'intérieur des théâtres ont dû contribuer à renforcer le sentiment de cohésion des communautés civiques cariennes comme ailleurs dans le monde $\operatorname{grec}^{61}$.

C'est aussi dans les théâtres que sont proclamés les honneurs décernés aux bienfaiteurs des cités selon des modalités caractéristiques des poleis hellénistiques ${ }^{62}$. Le décret honorifique de Théangéla pour Minniôn d'Antioche l'énonce ainsi (vers la fin du III $^{\mathrm{e}}$ et

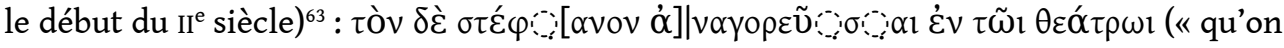
proclame la couronne dans le théâtre »). Compris dans leur dimension politique et culturelle, les théâtres paraissent donc étroitement liés au statut de polis ${ }^{64}$, et la présence ou non de théâtres dans certaines régions peut être considérée comme le reflet de différents niveaux d'hellénisation ${ }^{65}$. La poliadisation y paraît moins aboutie et la vie civique de ces communautés semble ne pas être rythmée par les représentations théâtrales. En effet, si du point de vue de la chronologie la Carie ne se démarque pas de l'Ionie voisine, en revanche, contrairement à l'Ionie, tous les centres civiques cariens ne paraissent pas avoir possédé de théâtre ${ }^{66}$. Pour autant, un établissement comme Kastabos, qui n'est pas une cité, peut en être pourvu ${ }^{67}$.

\section{Gymnases et palestres}

Les premières attestations de gymnases et de palestres datent de la fin du $\mathrm{IV}^{\mathrm{e}}$ siècle (Mylasa) et du III $^{\mathrm{e}}$ siècle, quand un gymnase est restauré à Halicarnasse ${ }^{68}$. Elles sont à replacer dans le contexte de l'essor de la paideia et de la diffusion des pratiques gymniques et de l'éphébie dans le monde gre ${ }^{69}$, la Carie ne présentant pas d'originalité particulière en terme de chronologie (tableau $3^{70}$ ). 
Tableau 3. - Gymnases et palestres de Carie.

\begin{tabular}{|c|c|c|c|}
\hline Ville & Épigraphie & Archéologie & Littérature \\
\hline Alabanda & & & $\begin{array}{l}\text { G: }: 1^{\text {re }} \text { moitié du I Ier s. ? } \\
\text { (Vitr. VII, } 5,6)\end{array}$ \\
\hline (Alinda) & G : gymnasiarque, ${ }^{\text {er }}$ s. $\left(S E G 63,2012, n^{\circ} 839\right)$ & & \\
\hline (Bargasa) & $\begin{array}{l}\text { G : gymnasiarque, } \mathrm{II}^{\circ} \mathrm{s} \text {. (Debord \& Varinlioğlu, } \\
2010, \mathrm{n}^{\circ} 5 \text { ) }\end{array}$ & & \\
\hline (Bargylia) & $\begin{array}{l}\text { G : gymnasiarque, dernier tiers du II } \mathrm{s} \text {. (SEG 50, } \\
\left.2000, \mathrm{n}^{\circ} 1101,1.18-19\right)\end{array}$ & & \\
\hline (Caunos) & G : gymnasiarque, HL (Marek, 2006, $\mathrm{n}^{\circ} 41$ ) & & \\
\hline Cnide & $\begin{array}{l}\mathrm{P}: \text { après } 282 \text { (IKnidos } 301) \\
\text { (G : fin de l'époque HL, catalogue de vainqueurs } \\
\left.\left(S E G 44,1994, \mathrm{n}^{\circ} 902\right)\right)\end{array}$ & G ?:c. 300 & \\
\hline \multirow[t]{2}{*}{ Halicarnasse } & G, P : III' s. (Isager, 2004, $\mathrm{n}^{\circ}$ 1) & & \\
\hline & G : III' s. : Philippeion (Migeotte, 1984, $\mathrm{n}^{\circ} 102$ ) & & \\
\hline (Héraclée du Latmos) & $\begin{array}{l}\text { (G : gymnasiarque : haute époque hellénistique } \\
\left.\text { (Haussoullier, 1899, } \mathrm{n}^{\circ} 7\right) \text {; fourniture d'huile : } \\
\text { entre } 196 \text { et } 193(S E G \text { 37, } 859 \text { (bloc I, 1. 8-11 et } \\
\text { bloc III, 1. 3-4)) }\end{array}$ & & \\
\hline Hydai (?) & $\begin{array}{l}\text { G, P : tardo-hellénistique (IMylasa 909): } \\
\text { provenance douteuse }\end{array}$ & & \\
\hline \multirow[t]{3}{*}{ Iasos } & (G : gymnasiarque : années 170 (IIasos 250)) & & \\
\hline & G: $: \mathrm{I}^{\mathrm{er}} \mathrm{s}$. (IIasos 98) : Ptolemaieion & & \\
\hline & G : HL (IIasos 93) : Antiocheion & & \\
\hline Kéramos & (G : gymnasiarque : HL (IKeramos $8,1.2)$ & P ?:HL & \\
\hline (Mobôlla) & $\begin{array}{l}\text { (G : gymnasiarque : } 1^{\text {ro }} \text { moitié du I }{ }^{\text {or }} \mathrm{s} . \\
(H T C 64,65))\end{array}$ & & \\
\hline Mylasa & $\begin{array}{l}\text { G, P : c. } 317 \text { (IMylasa } 21) \\
\left.\text { G: } \text { II }^{-}-I^{\text {er }} \text { s. (IMylasa } 137\right)\end{array}$ & & \\
\hline Nysa & G, A : I Ir s. (Str. XIV.1.43) & & \\
\hline Stratonicée de Carie & \multicolumn{2}{|c|}{ G : $2^{\mathrm{e}}$ quart du II ${ }^{\circ}$ s. (architrave $: \mathrm{II}^{\mathrm{e}}-\mathrm{I}^{\mathrm{er}} \mathrm{s}$. (Mert, 2008, 156-157)) } & \\
\hline Théangéla & P : HL (Robert, 1936, 65) & & \\
\hline Tralles & $\begin{array}{l}\text { (G : concours gymniques et éphèbes : fin IV } \mathrm{IV}^{\mathrm{c}}- \\
\mathrm{III}^{\mathrm{e}} \mathrm{s} .(\text { ITralleis } 106) \text {; gymnasiarque : } \mathrm{II}^{\mathrm{e}}-\mathrm{I}^{\mathrm{Ir}} \mathrm{s} \text {. } \\
(\text { ITralleis } 107))\end{array}$ & & $\begin{array}{l}\text { G : HL } \\
\text { (Str. XII, 8, 18) } \\
\text { S : HL (Vitr. V, 9, 1) }\end{array}$ \\
\hline
\end{tabular}

Le seul gymnase d'époque hellénistique mis au jour en Carie se trouve à Stratonicée. Il est situé dans la partie ouest de la ville, à proximité des remparts. De très grandes dimensions (plus de $18000 \mathrm{~m}^{2}$ ), il doit avoir été construit dans le deuxième quart du $\mathrm{II}^{\mathrm{e}}$ siècle ${ }^{71}$. Le long de son côté nord, une rangée de pièces comprend une exèdre semicirculaire centrale flanquée de quatre pièces rectangulaires (fig. 6). 
Fig. 6 - Gymnase de Stratonicée de Carie : l'exèdre centrale.

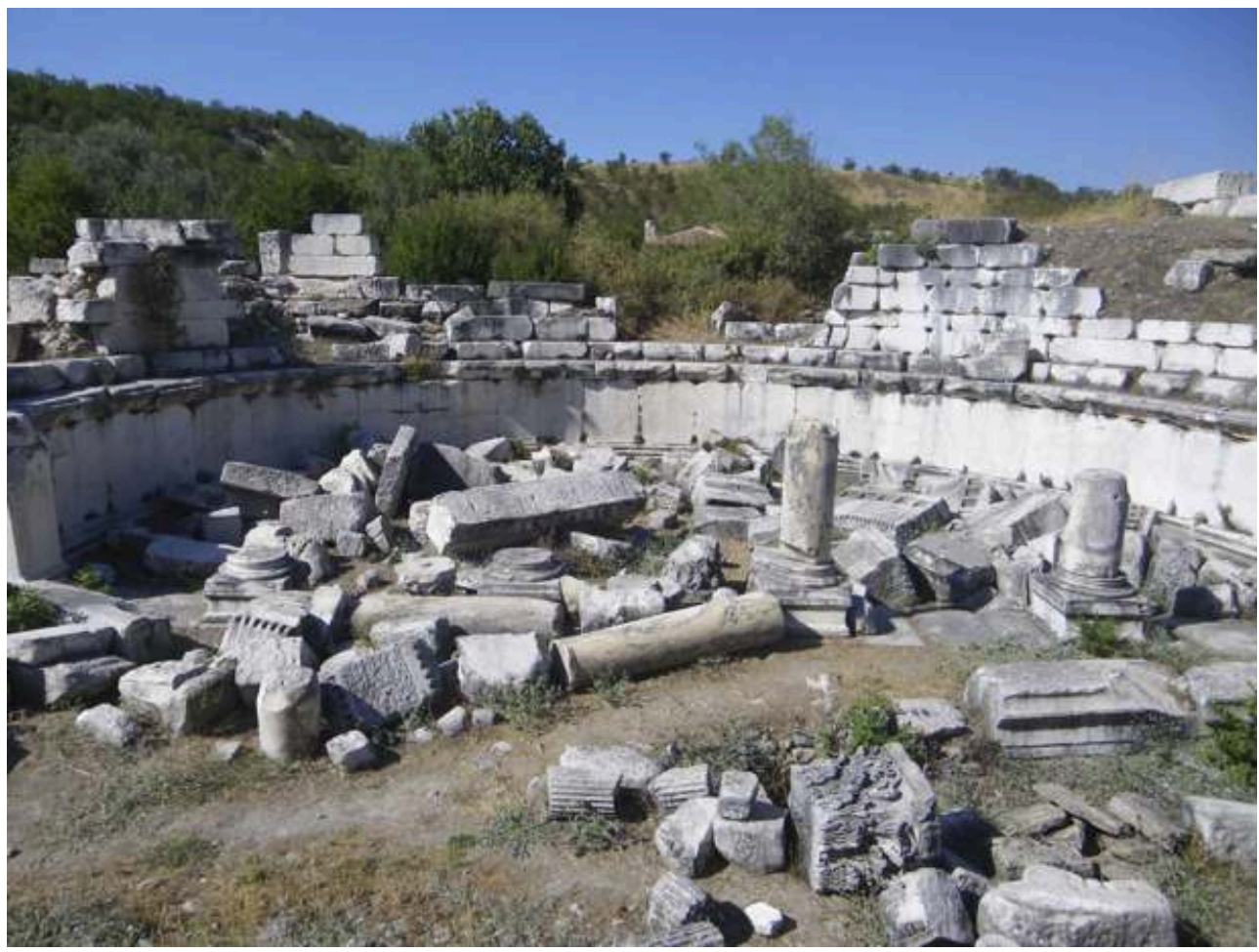

Au sud, la palestre devait occuper l'essentiel de l'édifice. L'ensemble est en marbre blanc et paraît avoir été pour partie au moins financé par la cité ${ }^{72}$. À Mylasa, le gymnase est restauré vers la même époque : un individu y est honoré pour avoir "restauré le

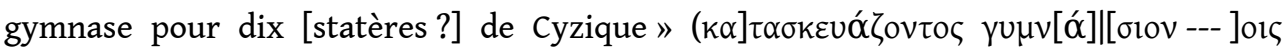

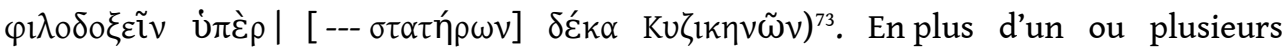
gymnases, certaines cités possèdent aussi une palestre distincte du gymnase. Au III siècle, en attendant la restauration du gymnase appelé Philippeion, les néoi et les paides d'Halicarnasse s'entraînent dans la «palestre des enfants" censée accueillir uniquement les paides en temps normal ${ }^{74}$. En Carie intérieure, les premières mentions de gymnases ou de gymnasiarques apparaissent aux $\mathrm{II}^{\mathrm{e}}-\mathrm{I}^{\mathrm{er}}$ siècles: à Bargasa, un gymnasiarque est connu au $\mathrm{II}^{\mathrm{e}}$ siècle et à Mobôlla, deux dédicaces d'éphébarques et de gymnasiarques datent de la première moitié $d u$ irr ${ }^{\text {er }}$ siècle ${ }^{75}$. Il est probable que ces cités possèdent un gymnase, mais la mention d'un gymnasiarque ou d'un éphébarque ne constitue pas une preuve de son existence, car les entraînements pouvaient se dérouler dans des lieux sommairement aménagés ${ }^{76}$. Les gymnases sont généralement réservés aux citoyens afin de préparer les jeunes hommes au maniement des armes dans le but de contribuer à la défense de la cité ${ }^{77}$. Comme ailleurs dans le monde grec, la population masculine des cités cariennes est divisée en classes d'âge, parmi lesquelles les paides, les éphèbes et les néoi fréquentent le gymnase ${ }^{78}$. Ainsi l'adoption du modèle politique de la cité grecque s'accompagne-t-elle de pratiques sociales nouvelles transformant probablement l'organisation de la défense des communautés qui ont pu trouver là un moyen plus efficace de se protéger. Les gymnases servent aussi de cadre à la formation intellectuelle et culturelle des jeunes dans les pièces qui bordent la palestre, comme l'exèdre du gymnase de Stratonicée de $\mathrm{Carie}^{79}$. Ainsi l'édification de gymnases révèle-telle le souci des cités d'éduquer et de former les jeunes gens à une certaine culture et à des pratiques qui doivent leur être utiles dans leur vie de citoyens. À partir de l'époque 
hellénistique tardive, ce type de bâtiment prend une place nouvelle dans la vie de la cité, devenant une sorte de "seconde agora ${ }^{80}$ ». Il abrite des cultes spécifiques (Hermès et Héraclès, héros locaux, évergètes, souverains ${ }^{81}$ et peut être le cadre de banquets ${ }^{82}$, tandis que certains bienfaiteurs peuvent y être honorés ${ }^{83}$.

Cette place grandissante du gymnase dans la vie civique a pu inciter les cités à se doter d'un gymnase à partir du $\mathrm{II}^{\mathrm{e}}$ siècle. La Carie s'inscrit dans un phénomène qui caractérise les cités de l'ensemble du monde grec à l'époque hellénistique ${ }^{84}$, même si l'apparition des gymnases y est un peu plus tardive qu'en Grèce. Celle-ci y est toutefois plus précoce que dans les régions d'Asie Mineure intérieure et orientale où les processus d'hellénisation et de poliadisation sont plus tardifs ${ }^{85}$. Le gymnase étant étroitement lié à la culture de la polis ${ }^{86}$, il apparaît là où les communautés adoptent le modèle de la cité grecque. En ce sens, comme ailleurs dans l'Orient grec, en Asie Mineure le gymnase apparaît aussi comme un marqueur de l'hellénisme ${ }^{87}$.

\section{Chronologie : le rythme des transformations urbaines}

28 L'établissement d'une chronologie des transformations urbaines est tributaire d'une documentation archéologique et épigraphique où les datations précises et fiables sont rares. Le rythme de la fabrique urbaine varie au cours de l'époque hellénistique, mais les constructions ne s'interrompent pas, y compris lors de périodes économiquement plus difficiles comme la première moitié $d u{ }^{\text {er }}$ siècle. Les premières attestations épigraphiques et les premiers vestiges archéologiques appartiennent à la fin du $\mathrm{IV}^{\mathrm{e}}$ et au début $\mathrm{du}$ III $^{\mathrm{e}}$ siècle, comme le théâtre de Cnide, le gymnase de Mylasa ou le bouleutérion d'Halicarnasse ${ }^{88}$. Par la suite, le $\mathrm{II}^{\mathrm{e}}$ siècle constitue un moment décisif dans la monumentalisation des centres urbains avec l'édification de théâtres (Bargasa), de sanctuaires (Cnide, Kodapè, Iasos) et de stoai (Héraclée du Latmos, Mylasa) ${ }^{89}$. C'est une période de prospérité économique dont bénéficient les centres urbains ${ }^{90}$. La deuxième moitié du II siècle constitue un temps d'effacement des dominations royales, ce qui n'a pas nécessairement pénalisé les cités du point de vue des financements. Au contraire, disposant d'une plus grande liberté financière, elles peuvent aussi solliciter d'autres bienfaiteurs qui continuent de soutenir l'activité de construction ${ }^{91}$.

\section{Les modalités de financement: des pratiques grecques au service de la fabrique urbaine}

La documentation épigraphique permet de saisir les modalités de financement des édifices qui composent peu à peu un paysage urbain grec en pays carien. À l'époque hellénistique, les cités peuvent financer les travaux de construction ou de restauration sur leurs fonds propres ou mobiliser les différents ressorts de l'évergétisme: souscriptions et emprunts publics, initiatives individuelles et de manière exceptionnelle soutien royal ${ }^{92}$. Alors qu'elles choisissent de bâtir des édifices destinés à abriter une vie civique grecque, les communautés adoptent aussi des pratiques grecques qui facilitent la mise en œuvre de leurs projets architecturaux. Le processus décisionnel aboutissant à la construction d'un édifice public est inconnu avant l'époque hellénistique en Carie. Ensuite, on retrouve les mêmes pratiques qu'ailleurs dans le 
monde grec, y compris dans de très petites communautés. Dans le cas des gymnases, le bâtiment peut à l'origine avoir été construit par un particulier puis être utilisé comme bâtiment public. Ainsi, à Mylasa, un certain Nicanor fait aménager une palestre et un gymnase au début du III $^{\mathrm{e}}$ siècle ${ }^{93}$. Mais les initiatives civiques doivent être plus fréquentes et les cités doivent trouver les moyens de financer les constructions et leur entretien. Les souscriptions publiques (épidoseis) appartiennent à l'éventail des solutions dont disposent les cités pour trouver des sources de financement pour leurs édifices publics. À Halicarnasse, le gymnase restauré dans la première moitié du $\mathrm{III}^{\mathrm{e}}$ siècle est financé par une souscription et un emprunt ${ }^{94}$. Les souscriptions publiques constituent une pratique grecque très répandue pour financer des constructions, $y$ compris dans des communautés qui présentent une organisation politique typiquement carienne. À Pisyè, la cité (dèmos) des Pisyètai et des Pladaseis unis aux Pisyètai lance une souscription publique pour financer la construction de néôria probablement situés dans la baie d'Akbük qui constitue l'accès à la mer de la cité (à l'est de Kéramos) ${ }^{95}$. Pisyè est composée d'une dizaine de communautés dont les ethniques sont conservés dans la liste des souscripteurs ${ }^{96}$. L'épidosis existe aussi dans des communautés ayant perdu le statut de polis après leur intégration dans la Pérée rhodienne comme le koinon des Tarmianoi (dont le centre se situe à Mobôlla) qui organise une souscription entre 225 et $150^{97}$.

Les cités recourent plus rarement à l'emprunt qui permet de disposer des liquidités ${ }^{98}$ qui leur font souvent défaut ${ }^{99}$. Au III $^{\mathrm{e}}$ siècle à Halicarnasse, le bouleutérion et une stoa sont au moins partiellement financés de cette manière ${ }^{100}$. Le décret organisant l'emprunt sans intérêt destiné à financer la stoa prévoit plusieurs hypothèques pour garantir les prêts ${ }^{101}$. Elles doivent représenter des sommes très importantes: des fonds déjà hypothéqués pour rembourser les prêts destinés à construire le bouleutérion, les revenus issus de la taxe du cinquantième et du "greffe des serments ", un talent provenant de l'oikonomia ${ }^{102}$, et le produit de la vente des matériaux de l'ancien portique. Les élites des cités cariennes contribuent aussi à la transformation de l'espace urbain par leurs évergésies qui financent tout ou partie d'un édifice. Toute la Carie et tous les types de cités paraissent concernés, quelle que soit leur taille. Comme ailleurs dans le sud-ouest micrasiatique, c'est au $\mathrm{II}^{\mathrm{e}}$ siècle que la pratique se répand et laisse des traces dans la documentation épigraphique. Les édifices financés reçoivent les activités caractéristiques des cités grecques: sièges des institutions civiques $^{103}$, théâtre ${ }^{104}$, gymnase $^{105}$, ou stoa ${ }^{106}$, élément caractéristique du paysage urbain grec et devenu indispensable à l'agora au cours de l'époque hellénistique.

Ces actes d'évergétisme d'ampleur variable sont intégrés à la mémoire civique par le vote de décrets honorifiques selon les modalités habituelles aux cités grecques adaptées aux spécificités locales. Ainsi à Mylasa, la tribu des Ortôkondeis remercie Limnaios fils d'Ouliades pour sa contribution à la construction d'une stoa sur l'agora décidée par la

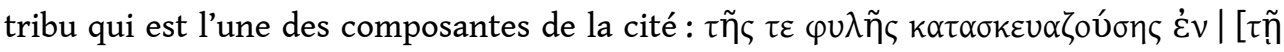

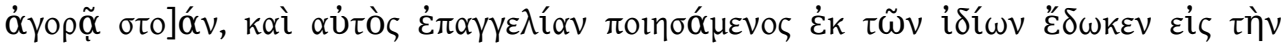

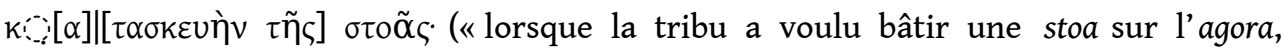
il a tenu sa promesse en contribuant sur ses biens à la construction de la stoa $[. .] ».)^{107}$. Les bienfaiteurs pratiquent aussi la dédicace qui associe durablement leur nom à l'édifice offert tout en prenant place dans l'espace urbain, comme l'agonothète Moschos qui embellit le théâtre d'Halicarnasse ${ }^{108}$ :

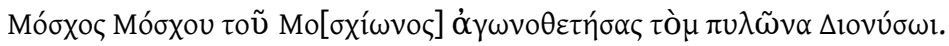


«Moschos, fils de Moschos, fils de Moschiôn, ancien agonothète, [a consacré]

la porte à Dionysos. »

Dans certaines cités cariennes, l'originalité est qu'en plus de sa famille le donateur associe sa tribu à son don, puisque traditionnellement les habitants de ces cités peuvent se désigner aussi de cette manière. Ainsi à Hyllarima, Anthènor a offert 1000 drachmes pour la construction d'une stoa. Il se désigne comme fils de Ménédènos, Tarmèsseus ${ }^{109}$. À l'inverse, Aristodèmos fils de Dioclès ne mentionne pas sa tribu dans sa dédicace du proskènion du théatre de cette même ville ${ }^{110}$. La cohabitation des deux pratiques dans une même cité $\mathrm{au} \mathrm{II}{ }^{\mathrm{e}}$ ou au début du $\mathrm{I}^{\mathrm{er}}$ siècle montre que les individus opèrent des choix différents relevant de stratégies de distinction personnelles, le premier conservant cette spécificité carienne qu'est la tribu et le second ne laissant rien voir de son identité carienne dans sa dédicace.

Les Cariens, peuvent aussi intégrer la pratique grecque de l'évergétisme dans l'organisation traditionnelle du koinon. À Kastabos, les inscriptions montrent que certains membres du koinon des Chersonésiotes ont contribué à la construction du sanctuaire ${ }^{111}$ :

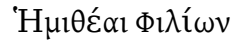

$\Phi \iota \lambda \omega ́ v \delta \alpha{ }^{\prime} Y \gamma \alpha \sigma \varepsilon \dot{\varsigma} \varsigma$

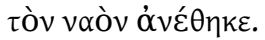

« Philiôn, fils de Philôndas, Hygaseus, a consacré le temple à Hémithéa. »

Ainsi, non seulement les Chersonésiotes ont recours à l'évergétisme pour la monumentalisation de leur sanctuaire commun, mais ils pratiquent eux aussi la dédicace architecturale, et ce dès le tout début de l'époque hellénistique.

L'évergétisme royal est plus rare en Carie, où comme ailleurs dans l'Asie Mineure hellénistique, les rois sont peu présents dans la documentation épigraphique ${ }^{112}$. Il peut s'agir d'un secours ponctuel aidant à la restauration d'édifices ou au relèvement de la ville après des destructions. À Iasos, l'intervention d'Antiochos III en faveur de l'épanorthôsis de la ville, littéralement son redressement, fait suite à un séisme qui frappe durement la ville en $199-198^{113}$. Elle se conjugue à l'action d'évergètes iasiens dont l'implication dans la restauration du théâtre et d'autres édifices a pu s'étaler sur quelques décennies ${ }^{114}$. L'implication royale paraît toutefois en retrait par rapport aux initiatives civiques. Cela doit relever d'un choix des Iasiens qui, tout en étant capables de solliciter un souverain, ne mettent pas en avant son action en faveur de la cité dans ce domaine (contrairement aux bienfaits politiques), soit dans le but de mettre en avant leur autonomie, soit parce que la domination séleucide ayant été brève à Iasos ${ }^{115}$, le soutien royal a pu être très ponctuel. Dans le cas de fondations royales, le rôle des souverains dans l'établissement de la trame urbaine a dû être déterminant et la construction des premiers édifices publics a pu être lancée dès les premiers temps de la ville nouvelle, même s'il ne faut pas surévaluer la capacité des fondateurs à aménager une ville ex-nihilo ${ }^{116}$. À Latmos, devenue plus tard Héraclée du Latmos, ce qui relèverait de l'initiative du satrape Asandros, à l'origine du synœcisme entre Latmos et sa voisine Pidasa, ne peut être déterminé ${ }^{117}$. À Stratonicée de Carie, Strabon rapporte que la ville « a été ornée par les rois de coûteux édifices » entre le milieu du III et le troisième tiers $\mathrm{du} \mathrm{II}^{\mathrm{e}}$ siècle ${ }^{118}$. Cette fondation séleucide, issue d'un synœcisme de cités cariennes complété par un apport de population macédonienne ${ }^{119}$, devait contribuer au contrôle 
royal de cette riche région et constituait un relais du prestige séleucide en Carie grâce à ses édifices publics.

$\mathrm{Au}$ total les financements paraissent le plus souvent le fait des communautés et de certains individus fortunés. La faible présence des souverains est frappante et constitue une caractéristique de l'époque hellénistique où les rois paraissent peu participer aux transformations urbaines. Cela peut refléter une réalité ou correspondre à un choix des souverains de se montrer discrets dans leurs interventions urbanistiques afin de conforter les apparences de l'autonomie des cités.

\section{Conclusion}

L'examen des constructions d'édifices politiques, de théâtres et de gymnases a montré que la constitution d'un paysage urbain de type grec est étroitement liée au phénomène politique et culturel de la diffusion et de l'affirmation des institutions civiques grecques. Au-delà du modèle de la cité grecque, l'adoption d'un fonctionnement démocratique qui caractérise la majorité des communautés à l'époque hellénistique a dû favoriser les transformations urbaines, comme cela a pu être démontré dans l'Athènes classique ${ }^{120}$. L'une des raisons de la construction d'édifices publics, en particulier ceux qui sont le siège des différentes institutions politiques et administratives de la cité, est qu'il existe une intense vie civique tout au long de l'époque hellénistique. De même, les communautés traduisent dans la pierre l'importance des pratiques culturelles qui prennent place dans les thêâtres et dans les gymnases. Ces édifices sont présents dans les cités issues de fondations royales où ils ont été amenés par les populations gréco-macédoniennes venues s'y installer. Ils se trouvent tout autant et aux mêmes époques dans les communautés où l'essentiel de la population paraît être d'origine carienne. En ce qui concerne les modalités de financement, les communautés ont mis en œuvre des pratiques financières grecques qui ont favorisé la constitution progressive d'un paysage urbain caractéristique d'une cité grecque. Toutefois, moyens de financement et édifices ne sont pas réservés aux poleis puisque les koina, qui sont un mode d'organisation politique très répandu en Carie, recourent aux mêmes outils et peuvent parfois construire les mêmes édifices que les poleis de la région. Cela révèle une forme de sélection des éléments grecs par les communautés cariennes, qui ont adapté certaines formes architecturales à leurs spécificités locales. Comme l'ont montré d'autres études portant sur les discours artistiques ou épigraphiques, les Cariens ont dû adopter certains éléments culturels grecs parce qu'ils les ont trouvés efficaces ${ }^{121}$. En l'occurrence, les institutions civiques de type grec ont ainsi favorisé la constitution d'un paysage urbain de type grec.

Le rythme de construction diffère généralement en fonction de la situation géographique des communautés, le littoral constituant l'un des principaux espaces de pénétration de l'hellénisme. Le démarrage est plus précoce dans les cités côtières où les attestations épigraphiques apparaissent souvent plus tôt qu'à l'intérieur, dès la fin du $\mathrm{IV}^{\mathrm{e}}$ et le début $\mathrm{du} \mathrm{III}^{\mathrm{e}}$ siècle, pour les premières, puis assez massivement pour l'ensemble de la région au $\mathrm{II}^{\mathrm{e}}$ siècle. Cette généralisation du mouvement de construction ne s'explique pas uniquement par les progrès de la diffusion du modèle de la cité grecque. Il est aussi lié au contexte politique régional propre à l'Asie Mineure après la paix d'Apamée et à une situation économique favorable qui a permis aux cités de disposer des moyens nécessaires à la réalisation de ces projets urbains. Néanmoins, certaines 
cités cariennes ne connaissent un développement architectural qu'à la toute fin de l'époque hellénistique et au début de l'époque augustéenne, dans les dernières décennies $\mathrm{du} \mathrm{I}^{\mathrm{er}}$ siècle. Un phénomène similaire caractérise certains centres urbains des régions proches de la Carie, où la pénétration du modèle de la cité grecque et des pratiques culturelles et sociales qui l'accompagne est un peu plus tardif ${ }^{122}$. Les rythmes de développement propres à chaque communauté doivent aussi être pris en considération. Ainsi dans la vallée du Méandre, à Nysa, le gérontikon, le gymnase et le théâtre sont construits dans la deuxième moitié du $\mathrm{e}^{\mathrm{er}}$ siècle, tandis qu'à Aphrodisias l'épanouissement architectural commence sous le Principat. Dans ces cités comme dans de nombreuses autres communautés de Carie, l'époque impériale constitue un nouveau temps de leur essor urbain marqué par les transformations sociales et les relations nouvelles avec Rome ${ }^{123}$.

\section{BIBLIOGRAPHIE}

\section{Abréviations}

- $A D=$ Arkeoloji Dergisi.

- HTC = Debord \& Varinlioğlu (2001).

- IK, 22.1-Stratonikeia = şahin (1982).

- IK, 24.1-Smyrne = Petzl (1987).

- IK, 28.1-Iasos = Blümel (1985a).

- IK, 28.2-Iasos = Blümel (1985b).

- IK, 30-Keramos = Varinlioğlu (1986).

- IK, 34-Mylasa = Blümel (1987a).

- IK, 35-Mylasa = Blümel (1987b).

- IK, 36.1-Tralleis = Poljakov (1989).

- IK, 41-Knidos = Blümel (1992).

- IK, 68-Stratonikeia = Şahin (2010).

- IKaunos = Marek (2006).

- KST = Kazı Sonuçları Toplantısı.

- $L B W=$ Le Bas \& Waddington ([1870] 1972).

ADIEGO Ignasi-Xavier, « Unity and Diversity in the Carian Alphabet », dans P. Brun, L. Cavalier, K. Konuk \& F. Prost (éd.), Euploia. La Lycie et la Carie antiques, Bordeaux, Ausonius, 2013, p. 17-28.

BANKEL Hansgeorg, « Knidos. Der hellenistische Rundtempel und sein Altar. Vorbericht », AA, 1997, p. 51-71.

BANKEL Hansgeorg, « Versatzmarken am Propylon des heilgitums für Apollon Karneios in Knidos ", dans M. Bachmann (éd.), Bautechnik im antiken und vorantiken Kleinasien (Internationale Konferenz, 13.-16. Juni 2007 in Istanbul, Byzas), Istanbul, Ege Yayinlari, 2009, p. 323-341.

BERTI Fede \& DELRIEUX Fabrice, « Un luogo di vendita dell'età medio-imperiale nell'agora di Iasos ", dans V. Chankowski \& P. Karvonis (éd.), Tout vendre, tout acheter. Structures et équipements des marchés antiques (Actes du colloque international d'Athènes, GDRI, EfA, Société archéologique d'Athènes, 16-19 juin 2009), Bordeaux, Ausonius, 2012, p. 105-118. 
BERTI Fede, MASTURZO Nicolò \& VITTORI Manuela, « New Studies of the Theatre at Iasos: 50 Years since the First Excavation », dans R. Frederiksen, E. R. Gerhard \& A. Sokolicek (éd.), The Architecture of the Ancient Greek Theatre (Acts of an International Conference at the Danish Institute at Athens, 27-30 January 2012), Monographs of the Danish Institute at Athens, Aarhus, Aarhus University Press, 2015, p. 131-147.

BLÜMEL Wolfgang, Die Inschriften von Iasos, IK, 28.1, Bonn, R. Habelt, 1985a.

BLÜMEL Wolfgang, Die Inschriften von Iasos, IK, 28.2, Bonn, R. Habelt, 1985b.

BLÜMEL Wolfgang (éd.), Die Inschriften von Mylasa, IK, 34-Mylasa, Bonn, R. Habelt, 1987a.

BLÜMEL Wolfgang (éd.), Die Inschriften von Mylasa, IK, 35-Mylasa, Bonn, R. Habelt, 1987b.

BLÜMEL Wolfgang (éd.), Die Inschriften von Knidos. Teil I, IK, 41-Knidos, Bonn, R. Habelt, 1992.

BLÜMEL Wolfgang, « Kopien A. Biliottis von Inschriften aus Halikarnassos, Bargylia, Keramos und Kos », AD, 2, 1994, p. 99-117.

BLÜMEL Wolfgang, « Neue Inschriften aus Karien II: Mylasa und Umgebung », EA, 37, 2004, p. 1-42.

BOYSAL Yusuf, « Die Bauten der hellenistischen Stadt Stratonikeia », dans Deutsches Archäologisches Institut (éd.), Akten des XIII. Internationalen Kongresses für Klassische Archäologie (Berlin, 1988), Berlin, Philipp von Zabern, 1990, p. 501-502.

BREMEN Riet van, « Neoi in Hellenistic Cities: Age Class, Institution, Association? », dans P. Fröhlich \& P. Hamon (éd.), Groupes et associations dans les cités grecques (III siècle av. J.-C.-II siècle apr. J.-C.), Genève, Droz, coll. « Hautes études du monde gréco-romain », 2013, p. 31-58.

BRESSON Alain, Inscriptions de la Pérée rhodienne (Pérée intégrée), Paris, Les Belles Lettres, 1991.

BRESSON Alain \& DESCAT Raymond, Les cités d'Asie Mineure occidentale au II siècle a.C., Bordeaux, Ausonius, 2001.

BRESSON Alain, « Italiens et Romains à Rhodes et à Caunos ", dans C. Müller \& C. Hasenohr (éd.), Les Italiens dans le monde grec. II siècle av. J.-C.-Ir siècle apr. J.-C., BCH supplément, Paris, 2002, p. $147-162$.

BRIANT Pierre, «L'Asie mineure en transition », dans P. Briant \& F. Joannes (éd.), La transition entre l'empire achéménide et les royaumes hellénistiques (vers 350-vers 300 av. J.-C.), Paris, De Boccard, 2006, p. 309-351.

BRUNS-ÖZGAN Christine (éd.), Knidos Ergebnisse der Ausgrabungen von 1996-2006, Istanbul, Ege Yayınları, 2013.

CHAISEMARTIN Nathalie de \& THEODORESCU Dinu, Le théâtre d'Aphrodisias : les structures scéniques, Wiesbaden, Reichert, 2017.

CHANKOWSKI Andrzej S., « L'entraînement militaire des éphèbes dans les cités grecques d'Asie Mineure à l'époque hellénistique : nécessité pratique ou tradition atrophiée ? », dans J.-C. Couvenhes \& H.-L. Fernoux (éd.), Les cités grecques et la guerre en Asie Mineure à l'époque hellénistique, Tours, Presses universitaires François Rabelais, 2004, p. 55-76.

CHANKOWSKI Andrzej S., L'éphébie hellénistique. Étude d'une institution civique dans les cités grecques des îles de la mer Égée et de l'Asie Mineure, Paris, De Boccard, 2011.

COUVENHES Jean-Christophe \& HELLER Anna, « Les transferts culturels dans le monde institutionnel des cités et des royaumes à l'époque hellénistique », dans J.-C. Couvenhes \& 
B. Legras (éd.), Transferts culturels et politique dans le monde hellénistique, Paris, Publications de la Sorbonne, 2006, p. 15-52.

COUVENHES Jean-Christophe \& LEGRAS Bernard, «Introduction », dans J.-C. Couvenhes \& B. Legras (éd.), Transferts culturels et politique dans le monde hellénistique, Paris, Publications de la Sorbonne, 2006, p. 5-11.

CROWTHER Charles, « Iasos in the Second Century II: The Chronology of the Theatre Lists ", BICS, 37, 1990, p. 143-152.

DAN Anca \& QUEYREL François, « Les concepts en sciences de l'antiquité : mode d'emploi. Chronique 2014 - Les transferts culturels », DHA, 40, 2014 (1), p. 239-305.

DE BERNARDI FERRERO Daria, Teatri Classici in Asia Minore, vol. 2 : Città di Pisidia, Licia e Caria, Rome, « L'Erma » di Bretschneider, 1969.

DEBORD Pierre, Aspects sociaux et économiques de la vie religieuse dans l'Anatolie gréco-romaine, Leyde, Brill, 1982.

DEBORD Pierre, «Questions stratonicéennes », dans A. Bresson \& R. Descat (éd.), Les cités d'Asie Mineure occidentale au II siècle a.C., Bordeaux, Ausonius, 2001, p. 157-172.

DEBORD Pierre, « Côte/intérieur : les acculturations de la Carie », PP, 341-345, 2005, p. 357-378.

DEBORD Pierre \& Ender VARINLIOĞLU (éd.), Les hautes terres de Carie, Bordeaux, Ausonius, 2001.

DEBORD Pierre \& VARINLIOĞLU Ender, Cités de Carie, Rennes, Presses universitaires de Rennes, 2010.

DEBORD Pierre \& VARINLIOĞLU Ender, Hyllarima de Carie, état de la question, Bordeaux, Ausonius, 2018.

DELORME Jean, Gymnasion. Étude sur les monuments consacrés à l'éducation en Grèce (des origines à l'Empire romain), Paris, De Boccard, 1960.

DELRIEUX Fabrice, « Les étrangers dans l'épigraphie iasienne du II siècle a.C. », dans A. Bresson \& R. Descat (éd.), Les cités d'Asie Mineure occidentale au II siècle a.C., Bordeaux, Ausonius, 2001, p. 137-155.

DINÇ Rafet, Tralleis Guide, Istanbul, Arkeoloji ve Sanat Yayınları, 2003.

DÖRPFELD Wilhelm, « Ausgrabungen im Theater von Magnesia am Maiandros. III. Das Theatergebäude », MDAI (A), XIX, 1894, p. 65-92.

DÖRPFELD Wilhelm \& REISCH Emil, Das griechische Theater, Aalen, Scientia, 1966 (Athènes, 1896).

DREYER Boris, « Die Neoi im hellenistischen Gymnasion », dans D. Kah \& P. Scholz (éd.), Das hellenistische Gymnasion, Berlin, De Gruyter, 2004, p. 211-231.

EDHEM Bey, « Fouilles d'Alabanda en Carie. Rapport sommaire sur la première campagne », CRAI, 49, 1905 (4), p. 443-459.

EHRHARDT Wolfgang, « Hellenistische Heiligtümer und Riten: Die westlichen Sakralbezirke in Knidos als Fallbeispiel », dans A. Matthaei \& M. Zimmermann (éd.), Stadtbilder im Hellenismus. Die hellenistische Polis als Lebensform, Francfort-sur-le-Main, Verlag Antike, 2009, p. 93-115.

EMME Burkhard, Peristyl und Polis. Entwicklung und Funktionen öffentlicher griechischer Hofanlagen, Berlin/Munich/Boston, De Gruyter, 2013.

FABIANI Roberta, « Un decreto ateniense riproposto a Iasos (IG II ${ }^{2} 3$ et Iasos 3926) », PP, 56, 2001, p. 69-100. 
FABIANI Roberta, I decreti onorari di Iasos. Cronologia e storia, Munich, C. H. Beck, 2015.

FROEHNER Wilhelm, Les inscriptions grecques du Louvre, Paris, Imprimeurs des Musées impériaux, 1865.

GAUTHIER Philippe, « Les cités hellénistiques : épigraphie et histoire des institutions et des

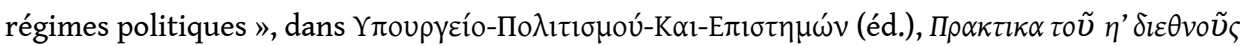

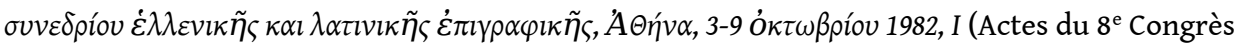
international d'épigraphie grecque et latine, Athènes, 3-9 octobre 1982, I), Athènes (= GAUTHIER 2011, 315-350), Musée épigraphique, 1984, p. 82-107.

GAUTHIER Philippe, Les cités grecques et leurs bienfaiteurs, Athènes/Paris, École française d'Athènes/De Boccard, 1985.

GAUTHIER Philippe, Nouvelles inscriptions de Sardes II, Genève, Droz, 1989.

GAUTHIER Philippe, Études d'histoire et d'institutions grecques. Choix d'écrits, Genève, Droz, 2011.

GAUTHIER Philippe \& HATZOPOULOS Miltiade B., La loi gymnasiarchique de Bérioa, Athènes/Paris, De Boccard, 1993.

GAUTHIER Philippe, « Notes sur le rôle du gymnase dans les cités hellénistiques », dans M. Wörrle \& P. Zanker (éd.), Stadtbild und Bürgerbild im Hellenismus, Munich, C. H. Beck Verlag (= GAUTHIER 2011, 531-550), 1995, p. 1-11.

GEHRKE Hans-Joachim, « Eine Bilanz: Die Entwicklung des Gymnasions zur Institution der Sozialisierung in der Polis », dans D. Kah \& P. Scholz (éd.), Das hellenistische Gymnasion, Berlin, De Gruyter, 2004, p. 413-419.

GOETTE Hans R., « Beobachtungen im Theater des Amphiareion von Oropos », AA, 110, 1995, p. 253-260.

GUIDOBONI Emmanuela (éd.), Catalogue of Ancient Earthquakes in the Mediterranean Area Up to the 10th Century, Rome, Istituto Nazionale di Geofisica, 1994.

HABICHT Christian, «Ist ein „Honoratiorenregime“ das Kennzeichen der Stadt im späteren Hellenismus? », dans M. Wörrle \& P. Zanker(éd.), Stadtbild und Bürgerbild im Hellenismus, Munich, C. H. Beck Verlag, 1995, p. 87-92.

HAUSSOULLIER Bernard, «Inscriptions d'Héraclée du Latmos », RPh, 23, 1899, p. 274-299.

HELD Winfried, « Heiligtümer und lokale Identität auf der karischen Chersones », dans P. Brun, L. Cavalier, K. Konuk \& F. Prost (éd.), Euploia. La Lycie et la Carie antiques, Bordeaux, Ausonius, 2013, p. 93-100.

HELD Winfried \& WILKENING-AUMANN Christine, « Vom karische Bund zur griechischen Polis. Archäologischer Survey in Bybassos und Kastabos auf der Karischen Chersones », dans A. Matthaei \& M. Zimmermann (éd.), Urbane Strukturen und bürgerliche Identität im Hellenismus. Die hellenistische Polis als Lebensform. Urbane Strukturen und bürgerliche Identität zwischen Tradition und Wandel, Francfort-sur-le-Main, Verlag Antike, 2015, p. 74-98.

HESBERG Henner von, « Das griechishe Gymnasion im 2. Jh. v. Chr. », dans M. Wörrle \& P. Zanker (éd.), Stadtbild und Bürgerbild im Hellenismus, Munich, C. H. Beck Verlag, 1995, p. 13-27.

HESBERG Henner von, « Hellenistische Theater - Zur Funktionalität der raüme und ihrer Bedeutung für die Polis ", dans A. Matthaei \& M. Zimmermann (éd.), Stadtbilder im Hellenismus. Die hellenistische Polis als Lebensform, Francfort-sur-le-Main, Verlag Antike, 2009, p. 276-303. 
HESBERG Henner von, « Theatergebäude und ihre Funktion in der Polis frühellenistischer Zeit ", dans A. Matthaei \& M. Zimmermann (éd.), Urbane Strukturen und bürgerliche Identität im Hellenismus. Die hellenistische Polis als Lebensform. Urbane Strukturen und bürgerliche Identität zwischen Tradition und Wandel, Francfort-sur-le-Main, Verlag Antike, 2015, p. 99-122.

HICKS Edward Lee, « On an Inscription at Cambridge: Boeck C. I. G. 106 », JHS, 74, 1881, p. 98-101. HOFF Ralf von den, « Hellenistische Gymnasia: Raumgestaltung und Raumfunktionen », dans A. Matthaei \& M. Zimmermann (éd.), Stadtbilder im Hellenismus. Die hellenistische Polis als Lebensform, Francfort-sur-le-Main, Verlag Antike, 2009, p. 245-275.

HORNBLOWER Simon, Mausolus, Oxford, Clarendon Press, 1982.

ISAGER Signe, « Halikarnassos and the Ptolemies I - Inscriptions on Public Buildings ", dans S. Isager \& P. Pedersen (éd.), The Salmakis Inscription and Hellenistic Halicarnassos, Odense, University Press of Southern Denmark, coll. « Halicarnassian Studies, 4 », 2004, p. 133-144.

ISLER Hans Peter, « Turchia », dans P. Ciancio Rossetto \& G. Pisani Sartorio (éd.), Teatri grecie romani. Alle origine del linguaggi rappresentato, vol. 3, Rome, Seat, 1994, p. 345-534.

ISLER Hans Peter, Antike Theaterbauten. Ein Handbuch, Vienne, Österreichische Akademie der Wissenschaften, 2017.

JENKINS Ian, The Lion of Knidos, Londres, British Museum Press, 2008.

JOHANNOWSKY Werner, « Osservazioni sul teatro di Iasos e su altri teatri in Caria », ASAA, 47-48, 1969-1970, p. 451-459.

KADIOĞLU Musa, Die Scaenae Frons des Theaters von Nysa am Mäander, Mayence-sur-le-Rhin, Philipp von Zabern, 2006.

KENZLER Ulf, Studien zur Entwicklung und Struktur der griechischen Agora in archaischer und klassischer Zeit, Francfort-sur-le-Main, Peter Lang, 1999.

KIZIL Abuzer, BRUN Patrice, CAPDETREY Laurent, DESCAT Raymond, FRÖHLICH Pierre \& KONUK Koray, « Pidasa et Asandros : une nouvelle inscription (321/320) », REA, 117, 2015, p. 371-409.

KIZIL Abuzer, KONUK Koray, ALEMDAR Sönmez, CAPDETREY Laurent, DESCAT Raymond, LAROCHE Didier, LE QUÉRÉ Enora, PROST Francis \& VERGNAUD Baptiste, « Eurômos : rapport préliminaire sur les travaux réalisés en 2016 », Anatolia Antiqua, 25, 2017, p. 161-185.

KOBES Jörn, « Teilnahmeklauseln beim Zugang zum Gymnasion », dans D. Kah \& P. Scholz (éd.), Das hellenistische Gymnasion, Berlin, De Gruyter, 2004, p. 237-245.

KOCKEL Valentin, « Bouleuteria. Architektonische Form und urbanistischer Kontext », dans M. Wörrle \& P. Zanker (éd.), Stadtbild und Bürgerbild im Hellenismus (Kolloquium, München, 24. bis 26. Juni 1993), Munich, C. H. Beck Verlag, 1995, p. 29-40.

KOLB Frank, « Le pouvoir politique et son cadre architectural en Lycie antique », dans P. Brun, L. Cavalier, K. Konuk \& F. Prost (éd.), Euploia. La Lycie et la Carie antiques, Bordeaux, Ausonius, 2013, p. 113-126.

KRISCHEN Fritz (éd.), Antike Rathaüser, Berlin, Gerb. Mann, 1941.

LAFON Xavier, MARC Jean-Yves \& SARTRE Maurice, « La ville antique », dans J.-L. Pinol (éd.), Histoire de l'Europe urbaine, t. 1 : De l'Antiquité au XVIII siècle, Paris, Seuil, 2003, p. 18-283.

LARGUINAT-TURBATTE Gabrièle, « Les premiers temps d'Arsinoeia-Ephèse. Étude d'une composition urbaine royale (début du III ${ }^{\mathrm{e}} \mathrm{s}$ ) », REA, 116, 2014 (2), p. 465-491. 
LARGUINAT-TURBATTE Gabrièle, « La place de l'architecture dans la description de l'Asie Mineure de Strabon », DHA, à paraître, p. 239-305.

LAUTER Hans, « Reisenotizen aus Karien », BJ, 171, 1971, p. 132-149.

LE BAS Philippe \& WADDINGTON Philippe Henry, Inscriptions grecques et latines recueillies en Asie Mineure, $2^{\mathrm{e}}$ éd., Hildesheim/New York, Georg Olms, 1972 (Paris, 1870).

LE GUEN Brigitte, « Théâtre et cités à l'époque hellénistique : "Mort de la cité" - "Mort du théâtre" ? ", REG, 108, 1995, p. 59-90.

LEVI Doro, « Iasos. Le campagne di scavo 1969-1970 », ASAA, 31-33, 1969-1970, p. 461-532.

LINDERS Tullia \& HELLSTRÖM Pontus (éd.), Architecture and Society in Hecatomnid Caria (Proceedings of the Upsalla Symposium, 1987), Upsalla, Acta Universitatis Upsaliensis, 1989.

MA John, « The Epigraphy of Hellenistic Asia Minor: A Survey of Recent Research (1992-1999) », AJA, 104, 2000, p. 95-121.

MADDOLI Gianfranco, « Nuovi testi da Iasos », PP, 316-317, 2001, p. 15-32.

MADDOLI Gianfranco, « Epigrafi di Iasos, Nuovi supplementi, I », PP, 62, 2007, p. 193-372.

MARCHESE Ronald T., The Lower Maeander Flood Plain, Oxford, British Archaeological Reports, 1986.

MAREK Christian, « Karien im Ersten Mithridatischen Krieg », dans P. Kneissl \& V. Losemann (éd.), Alte Geschichte und Wissenschaftsgeschichte. Festischrift für Karl Christ zum 65. Geburstag, Darmstadt, Wissenschaftliche Buchgesellschaft, 1988, p. 285-308.

MAREK Christian, Die Inschriften von Kaunos, Munich, C. H. Beck, 2006.

MASTURZO Nicolò, « Naiskos a edicola nell'Agorà di Iasos. Elementi per la definizione del tipo », Palladio, 8, 1995 (15), p. 5-14.

MEIER Ludwig, Die Finanzierung öffentlicher Bauten in der hellenistischen Polis, Berlin, Verlag Antike, 2012.

MERKELBACH Reinhold, « Ehrendekret für Alexandros aus Seleukeia-Tralles », ZPE, 17, 1975, p. $163-166$.

MERT Ibrahim Hakan, Untersuchungen zur hellenistischen und kaiserzeitlichen Bauornamentik von Stratonikeia, Tübingen, Ernst Wasmuth, 2008.

MIGEOTTE Léopold, L'emprunt public dans les cités grecques. Recueil des documents et analyse critique, Québec/Paris, Les Éditions du Sphynx/Les Belles Lettres, 1984.

MIGEOTTE Léopold, Les souscriptions publiques dans les cités grecques, Genève/Québec, Éditions du Sphynx/Librairie Droz, 1992.

MIGEOTTE Léopold, «La planification des dépenses publiques dans les cités hellénistiques », dans B. Virgilio (éd.), Studi ellenistici, Pise/Rome, Fabrizio Serra editore, 2006, p. 77-97.

MORETTI Jean-Charles, Théâtre et société dans la Grèce antique. Une archéologie des pratiques théâtrales, Paris, Librairie générale française, 2001.

NAUMANN Rudolf \& NAUMANN Friederike, Der Rundbau in Aezani mit dem Preisedikt des Diokletian und das Gebaude mit dem Edikt in Stratonikeia, Tübingen, Wasmuth, coll. « Istanbuler Mitteilungen, Beiheft $10 », 1973$.

NILSSON Martin P., Die hellenistische Schule, Munich, C. H. Beck, 1955. 
PAGA Jessica, « Deme Theaters in Attica and the Trittys System », Hesperia, 79, 2010 (3), p. 351-384.

PEDERSEN Poul, « The Ionian Renaissance and Some Aspects of Its Origin within the Field of Architecture and Planning ", dans J. Isager (éd.), Hekatomnid Caria and the Ionian Renaissance (Acts of the International Symposium at the Department of Greek and Roman Studies, Odense University, 28-29 November 1991), Odense, Odense University Press, coll. « Halikarnassian Studies, 1 », 1994, p. 11-35.

PEDERSEN Poul, « Reflections on the Ionian Renaissance in Greek Architecture and Its Historical Background », Hephaistos, 19/20, 2001/2002, p. 97-130.

PEDERSEN Poul \& ISAGER Signe, « The Theatre at Halikarnassos - and Some Thoughts on the Origin of the Semicircular Greek Theater. With an Appendix "The Inscriptions from the Theatre at Halikarnassos" ", dans R. Frederiksen, E. R. Gerhard \& A. Sokolicek (éd.), The Architecture of the Ancient Greek Theatre (Acts of an International Conference at the Danish Institute at Athens, 2730 January 2012), Monographs of the Danish Institute at Athens, Aarhus, Aarhus University Press, 2015, p. 293-317.

PESCHLOW-BINDOKAT Anneliese (éd.), Der Latmos. Eine unbekannte Gebirgslandschaft an der türkischen Westküste, Mainz am Rhein, Philipp von Zabern, 1996.

PESCHLOW-BINDOKAT Anneliese, Eine karische Gebirglandschaft. Heraklea am Latmos. Stadt und Umgebung, Ankara, Homer Kitabevi, 2005.

PETZL Georg (éd.), Die Inschriften von Smyrna, II 1, IK, 24.1-Smyrne, Bonn, Dr Rudolf Habelt GmbH, 1987.

PICKARD-CAMBRIDGE Arthur Wallace, The Theater of Dionysus in Athens, Oxford, Clarendon Press, 1966 (1956).

PIRAS Daniela, « Who Were the Karians in Hellenistic Times? The Evidence from Epichoric Language and Personal Names », dans R. V. Bremen \& J.-M. Carbon (éd.), Hellenistic Karia (Proceedings of the First International Conference on Hellenistic Karia, Oxford, 29 June-2 July 2006), Bordeaux, Ausonius, 2010, p. 217-233.

POLJAKOV Fjodor B. (éd.), Die Inschriften von Tralleis und Nysa, IK, 36.1-Tralleis, Bonn, Dr Rudolf Habelt GmbH, 1989.

PROST Francis, «Identité des peuples, identités des cités : l'exemple lycien », dans J.-M. Luce (éd.), Identités ethniques dans le monde grec antique, Pallas, 73, 2007, p. 99-114.

PROST Francis, « Retour au Mausolée et au Monument des Néréïdes. Identités ethniques et frontières culturelles en Lycie et en Carie ", dans P. Brun, L. Cavalier, K. Konuk \& F. Prost (éd.), Euploia. La Lycie et la Carie antiques, Bordeaux, Ausonius, 2013, p. 175-186.

RIVALLAND Anne-Sophie, Architecture et pouvoir : les villes de Pamphylie et de Pisidie, thèse de doctorat, Centre de recherches en histoire internationale et atlantique, 2014.

ROBERT Jeanne \& ROBERT Louis, La Carie, t. II : Le plateau de Tabai et ses environs, Paris, Librairie d'Amérique et d'Orient Adrien-Maisonneuve, 1954.

ROBERT Jeanne \& ROBERT Louis, Fouilles d'Amyzon en Carie, t. 1 : Exploration, histoire, monnaies et inscriptions, Paris, De Boccard, 1983.

ROBERT Jeanne \& ROBERT Louis, Claros I. Décrets hellénistiques, Paris, Éditions Recherches sur les civilisations, 1989. 
ROBERT Louis, Collection Froehner, vol. I : Inscriptions grecques, Paris, Éditions des Bibliothèques nationales, 1936.

ROBERT Louis, Études anatoliennes. Recherches sur les inscriptions grecques de l'Asie Mineure, Amsterdam, Adolf M. Hakkert, 1937.

ROBERT Louis, « Décrets de Smyrne pour des juges étrangers », Hellenica, 7, 1949, p. 170-188.

ROBERT Louis, « Recherches épigraphiques : V. Inscription de Lesbos », REA, 62, 1960a, p. 285-315 (= OMS II 801-831).

ROBERT Louis, « Décret d'Andros », Hellenica, 11-12, 1960b, p. 116-125.

ROSTOVTSEFF Michel Ivanovic, Histoire économique et sociale du monde hellénistique, Paris, Robert Laffont, 1989 (1941).

RUMSCHEID Jutta \& RUMSCHEID Frank, « Gladiatoren in Mylasa », AA, 2001, p. 115-136.

ŞAHIN Çetin (éd.), Die Inschriften von Stratonikeia, IK, 22.1-Stratonikeia, Bonn, Dr Rudolf Habelt, 1982.

ŞAHIN Çetin (éd.), The Inscriptions of Stratonikeia, IK, 68-Stratonikeia, Bonn, Dr Rudolf Habelt, 2010.

SARTRE Maurice, D’Alexandre à Zénobie. Histoire du Levant antique, IV siècle av. J.-C.-III siècle apr. J.-C., Paris, Fayard, 2001.

SAVALLI-LESTRADE Ivana, Les Philoi royaux dans l'Asie hellénistique, Genève, Librairie Droz, 1998.

SCHMIDT-DOUNAS Barbara, Geschenke erhalten die Freundschaft. Politik und Selbstdarstellung im Spiegel der Monumente, Berlin, Akademie Verlag, 2000.

SCHMITT-PANTEL Pauline, La cité au banquet. Histoire des repas publics dans les cités grecques, Rome, École française de Rome, 1992.

SERDAROĞLU Ümit, « Bautätigkeit in Anatolien unter der persischen Herrschaft », dans D. Papenfuss \& V. M. Strocka (éd.), Palast und Hütte. Beitrage zu Bauten und Wohnen im Altertum von Archäologen, Vor- und Frühgeschichtlern, Mayence, Philipp von Zabern, 1982, p. 347-356.

SÖĞÜT Bilal, « Stratonikeia 2011 yılı çalışmaları », KST, 34, 2012 (3), p. 45-58.

SÖĞÜT Bilal, « Stratonikeia 2012 yılı çalışmaları », KST, 35, 2013 (3), p. 448-464.

SPANU Marcello, Keramos di Caria. Storia e monumenti, Rome, «L'Erma » di Bretschneider, coll. «Studia Archaeologica, $89 », 1997$.

SPANU Marcello, «Il teatro ellenistico in Asia Minore. Aspetti funzionali, costruttivi e urbanistici », dans C. G. Malacrino \& E. Sorbo (éd.), Architetti, architettura e città nel Mediterraneo antico, Milan, Bruno Mondadori, 2007, p. 66-79.

VALOIS René, « Le théâtre de Tégée », BCH, 50, 1926, p. 135-173.

VARINLIOĞLU Ender (éd.), Die Inschriften von Keramos, Bonn, R. Habelt, 1986.

VARINLIOĞLU Ender, « East of Halicarnassus », dans S. Isager \& P. Pedersen (éd.), The Salmakis Inscription and Hellenistic Halicarnassos, Odense, University Press of Southern Denmark, coll. « Halicarnassian Studies, 4 », 2004, p. 125-131.

WÖRRLE Michael, « Inschriften von Herakleia am Latmos I : Antiochos III., Zeuxis und Herakleia », Chiron, 18, 1988, p. 422-476.

WÖRRLE Michael, « Inschriften von Herakleia am Latmos III. Der Synoikismos der Latmoi mit den Pidaseis ", Chiron, 33, 2003, p. 121-143. 
WULZINGER Karl, « Das Rathaus von Herakleia am Latmos », dans F. Krischen (éd.), Antike

Rathaüser, Berlin, Verlag Gerb. Mann., 1941, p. 22-33.

\section{NOTES}

1. Les illustrations (carte et photographies) sont de l'auteur.

2. Par exemple : Robert (1954); Debord \& Varinlioğlu (2001); Piras (2010); Adiego (2013).

3. Sauf précision contraire, toutes les dates s'entendent avant J.-C.

4. P. ex. à Bargasa, Orthosia et Hyllarima : cf. Debord \& Varinlioğlu (2010). Sur les difficultés de datation, voir infra n. 12.

5. Sur la poliadisation, voir Sartre (2001, 279-281) et Couvenhes \& Heller (2006). Sur la poliadisation en Carie, voir Debord $(2005,377)$. Dans l'est de la Carie l'hellénisation ne commence sans doute qu'au début de l'époque hellénistique. Sur le plateau de Tabai, voir Robert (1937, 338), Robert $(1954,377)$ et Marchese $(1986,169)$; Debord \& Varinlioğlu $(2010,166)$ sur l'hellénisation de Pisyè aux IV ${ }^{\mathrm{e}}$-III ${ }^{\mathrm{e}}$ siècles.

6. Le terme d'hellénisation, aujourd'hui débarrassé de toute hiérarchisation entre une culture grecque considérée comme dominante et une culture carienne considérée comme dominée (Dan \& Queyrel, 2014,245), peut être employé pour qualifier l'adoption de certains traits culturels grecs par les communautés cariennes. La notion de transfert culturel, plus neutre, n'évite pas nécessairement l'interprétation d'une relation inégale entre éléments culturels grecs et cariens : cf. Couvenhes \& Legras $(2006,8)$ et Prost $(2013,176)$. Enfin pour parler d'acculturation, il faut pouvoir déceler des caractéristiques cariennes dans les édifices de type grec, ce qui est rarement le cas. Une étude architecturale fine pourrait probablement permettre de dégager des spécificités locales dans les techniques de construction (extraction et taille des blocs, engins de levage, appareils mis en œuvre comme à Stratonicée de Carie). Ainsi, certains édifices comme l'autel du temple de Zeus Lepsynos à Eurômos présentent des mortaises en queue d'aronde destinées à recevoir des scellements en bois : cf. Laroche \& Alemdar, dans Kizil et al. $(2017,177)$. Le bouleutérion à gradins rectilignes est peut-être une forme micrasiatique (Notion, Héraclée du Latmos), mais ne peut être attribué à la Carie et demeure rare.

7. Debord $(2005,377)$.

8. Hornblower (1982). Sur l'architecture et l'urbanisme dans la Carie hécatomnide, voir Linders \& Hellström (1989). Sur la notion de «renaissance ionienne », voir Pedersen (1994) et Pedersen (2001/2002). Voir aussi Prost (2013, 175-176).

9. Cette "grécisation " est profonde dans les régions qu'ils dominent et plus tardive dans les espaces voisins. Elle se renforce à partir de la conquête d'Alexandre et dans les royaumes macédoniens qui lui succèdent, notamment avec la fondation de poleis : cf. Debord $(2005,377)$. Sur les fondations royales, voir infra n. 14.

10. Debord \& Varinlioğlu $(2010,345)$ : p. ex. le naos de Bargasa et l'agora d'Orthosia doivent être aménagés fin $\mathrm{II}^{\mathrm{e}}$-début $\mathrm{I}^{\mathrm{er}} \mathrm{s}$.

11. Pausanias, Description de la Grèce, $\mathrm{X}, 4,1$.

12. De nombreux édifices sont dans un bon état de conservation, mais ont rarement été fouillés. Les datations sont par conséquent le plus souvent imprécises et il est impossible de mener une étude exhaustive qui intègrerait l'ensemble des édifices hellénistiques des cités cariennes. Pour les mêmes raisons, il est difficile de proposer des datations pour la mise en œuvre d'une trame urbaine régulière caractéristique de l'urbanisme grec. Néanmoins les exemples convoqués ici permettent d'englober assez largement le phénomène de constitution d'un paysage urbain associé à celui de poliadisation. D'autre part, la documentation épigraphique abondante en Carie porte à la connaissance des historiens des édifices qui n'ont pas été mis au jour par les 
archéologues et fournit de nombreuses informations sur les pratiques institutionnelles entourant la fabrique urbaine (sur cette notion, voir infra n.13). Sur la pratique épigraphique en Asie Mineure à l'époque hellénistique, voir Ma (2000, 107-111) ; les décrets honorifiques constituent une part importante de la documentation. Enfin, les sources littéraires comportent peu de références à l'architecture et au paysage urbain, car les auteurs anciens paraissent s'en être désintéressés ; c'est par exemple le cas de Strabon : cf. Larguinat-Turbatte (à paraître).

13. Pour une mise au point sur la notion de fabrique urbaine, voir Larguinat-Turbatte (2014, 466-467, avec références).

14. À ce sujet, les fondations royales et les installations de colons macédoniens sont un facteur déterminant, soit dans les fondations mêmes, soit dans les communautés environnantes : cf. Debord (2005, 377-378) et Piras $(2010,232)$.

15. Dans les tableaux, les sources permettant de connaître l'existence d'un édifice sont abrégées ainsi : les attestations archéologiques sont notées « $\mathrm{A}$ », les attestations épigraphiques « $\mathrm{E}$ » et les attestations issues des sources littéraires « $\mathrm{L}$ ». Pour les datations, l'époque hellénistique est abrégée « HL », l'époque romaine « $\mathrm{R}$ ».

16. Naumann \& Naumann (1973, 67-79) ; Söğüt (2012, 48).

17. Sur Alabanda, voir Lauter $(1971,134-139)$ et Isler $(2017,41)$. Sur Héraclée du Latmos, voir Wulzinger $(1941,22-28)$ et Emme $\left(2013, n^{\circ} 33\right)$. Sur Stratonicée de Carie, voir Naumann \& Naumann $(1973,67-79)$, Boysal $(1990,502)$ et Mert $(2008,20)$ (fin de l'époque hellénistique). Pour les deux premiers bâtiments la datation au II $^{\mathrm{e}}$ siècle demeure approximative. Sur l'architecture des bouleutéria en général, voir Kockel (1995).

18. Isager (2004, $\left.\mathrm{n}^{\circ} 4 \mathrm{a}, 1.10\right)$; Fabiani $(2015,314,1.2)$; IK, 28.2-Iasos 252.

19. Appien, Mithridatique, 23. En 88, lors du massacre des Italiens, certains d'entre eux se réfugient auprès de l'autel d'Hestia, dans le bouleutérion de Caunos. Ils espèrent avoir la vie sauve mais sont tués par les Cauniens. Cf. Bresson (2002, 156-162).

20. Le premier abrite les réunions de l'assemblée, le second celles du conseil des anciens, la gérousia. Cf. Vitruve, De l'architecture, VII, 8, 5-7 ; Strabon, Géographie, XIV, 1, 43 (C 649).

21. Kizıl et al. (2015, 1. 13). L'hypothèse la plus probable est que les personnages honorés soient trois épimélètes (ibid., 383).

22. IK, 28.2-Iasos 608, 1. 31-32.

23. $I K$, 28.1-Iasos 82, 1. 27-28.

24. IK, 36.1-Tralleis 25, 1. 6-7. Sur l'identité d'Alexandros, voir : Merkelbach (1975) ; J. et L. Robert dans Bull. Ep $(1976,636)$.

25. IKaunos, 19, 1. 97-98.

26. IK, 28.2-Iasos 608, 1.31-32 ; Blümel (1994, $\left.\mathrm{n}^{\circ} 45,2-4\right)$. Le prytanée a pu être endommagé au cours de la guerre d'Aristonicos. Sur la cité de Bargylia pendant cette guerre, voir Marek $(1988,302)$ et Robert $(1989,30-31)$.

27. Sauf mention contraire, toutes les traductions sont de l'auteur.

28. IK, 41-Knidos 221, 1. 47-48, 1. 53 ; Isager (2004, $\left.\mathrm{n}^{\circ} 4 \mathrm{a}, 1.19\right)$.

29. IK, 28.1-Iasos 30, 1.12; IK, 28.1-Iasos 31, 1.4-5; IK, 28.2-Iasos 252, 1. 1 ; Maddoli (2007, $\left.\mathrm{n}^{\circ} 25 \mathrm{~A}, 1.6\right)$. L'archeion est vraisemblablement situé dans la partie sud de l'agora, à proximité du bouleutérion et de la stoa de Poséidon : cf. Fabiani (2001, 93 et 95).

30. IK, 28.1-Iasos 22, 1. 7. Sur le local lui-même, voir Levi (1969-1970, 493) et Berti \& Delrieux (2012, 107-108).

31. IK, 68-Stratonikeia 1505, 1. 4.

32. Sur Pidasa, voir Kizıl et al. $(2015,1.13)$.

33. Piras (2010).

34. Serdaroğlu $(1982,355)$.

35. Berti, Masturzo \& Vittori $(2015,141)$. 
36. Les références entre parenthèses concernent un indice épigraphique laissant penser qu'un théâtre existe dans la cité. Les références en gras correspondent à la construction de l'édifice ou à des travaux.

37. Sur Alinda, voir De Bernardi Ferrero $(1969,197)$. Sur Bargasa, voir Debord \& Varinlioğlu $(2010,149)$. Sur Eurômos, voir Isler $(1994,376)$ et Isler $(2017,285)$.

38. Porte ouest (II siècle) : cf. Pedersen \& Isager $(2015,310)$. Dédicace reproduite infra.

39. Hyllarima ( $\mathrm{II}^{\mathrm{e}}$ ou début du $\mathrm{I}^{\mathrm{er}}$ siècle) : cf. Debord \& Varinlioğlu (2018, $\left.\mathrm{n}^{\circ} 22\right)$. Stratonicée de Carie (II siècle), proskènion : cf. Mert (2008, PS. AF. 2) ; IK, 22.1-Stratonikeia 1013.

40. Robert (1937, 523), qui s'appuie sur Strabon, Géographie, XIV, 2, 25 (C 660). Johannowsky (1969-1970, 457) envisage aussi une construction du koilon lors de la fondation de la cité qu'il attribue à Antiochos I ${ }^{\text {er }}$. Sur la fondation de Stratonicée de Carie, voir Debord $(2001,157)$.

41. À titre de comparaison, le koilon du théâtre de Magnésie du Méandre mesure environ $75 \mathrm{~m}$ de diamètre : cf. Dörpfeld (1894, ca IV $\mathrm{IV}^{\mathrm{e}} \mathrm{II}{ }^{\mathrm{e}}$ s.) ; celui de Patara $84 \mathrm{~m}$ : cf. De Bernardi Ferrero (1969, 123-133, première moitié du $\mathrm{I}^{\mathrm{er}}$ siècle apr. J.-C.).

42. Il s'agit d'un appareil rectangulaire présentant une assise d'orthostates surmontée de parpaings en carreaux et de parpaings en panneresses visible dans le bouleutérion et le gymnase.

43. Mert $(2008,93$, n. 582). Les escaliers d'accès à la partie supérieure du koilon datent de l'époque impériale d'après Söğüt $(2013,455-457)$.

44. Les nombreux blocs conservés autorisent des restitutions relativement fiables.

45. Mert (2008, PS. AF. 2) ; IK, 22.1-Stratonikeia 1031. Sur les triglyphes, voir Mert $(2008,107)$.

46. IK, 22.1-Stratonikeia 1013.

47. Held \& Wilkening-Aumann $(2015,84)$. L'édifice qui n'a pas été fouillé n'est pas daté précisément, mais le site de Kastabos est abandonné à la fin de l'époque hellénistique : cf. Held $(2013,94)$.

48. Held $(2013,95)$; Held \& Wilkening-Aumann $(2015,86)$.

49. Un temple périptère est bâti par des architectes d'Halicarnasse : cf. Held (2013, 95-96) et Held \& Wilkening-Aumann (2015, 82-84).

50. Les Dionysies de Kéramos, connues par une inscription hellénistique (IK, 30-Keramos 9 , 1. 11-12), ne peuvent être formellement reliées au théâtre qui n'est pas précisément daté. Sur le théâtre, voir Isler $(2017,389)$.

51. À l'époque classique, les représentations dramatiques pouvaient se faire sur une estrade en bois ou dans des édifices avec gradins en bois : cf. Moretti $(2001,182)$. C'est probablement aussi le cas dans certaines villes à l'époque hellénistique avant la construction d'édifices en pierre.

52. Rumscheid \& Rumscheid (2001, 132-134) ; Isler (2017, 509-510).

53. Les théâtres en bois sont attestés en Attique jusqu'à l'époque classique : Thorikos, cf. Paga $\left(2010,355: \mathrm{VI}^{\mathrm{e}} \mathrm{s}.\right)$; théâtre de Dionysos à Athènes, cf. Dörpfeld \& Reisch ([1896] 1966, 28-29 : VI ${ }^{\mathrm{e}}$ $\mathrm{V}^{\mathrm{e}} \mathrm{s}$.) et Pickard-Cambridge ([1956] 1966, 11: début $\mathrm{V}^{\mathrm{e}} \mathrm{s}$.). Ils existent encore à l'époque hellénistique en Grèce (par exemple à Oropos : cf. Goette (1995); à Tégée le koilon est en bois jusqu'au II ${ }^{\mathrm{e}}$ siècle : cf. Tite-Live, Histoire romaine, XLI, 20 ; Valois (1926).

54. Sur Bargasa, voir Debord \& Varinlioğlu (2010, 145-152). Sur Orthosia, voir Debord \& Varinlioğlu (2010, 299-306). Sur le site de Taşyenice (région de Yerkesik, au nord d'Idyma et au sud-ouest de Mobôlla) qui pourrait être l'un des centres de la cité de Thèra, voir Debord \& Varinlioğlu $(2001,38)$.

55. Sur l'emploi de la langue carienne, voir Briant (2006, 322-326) et Piras (2010, 219-220). L'hellénisation n'en est pas moins profonde, comme le montre l'onomastique devenant majoritairement grecque à l'époque hellénistique : cf. Piras $(2010,232)$.

56. Le Guen $(1995,79)$.

57. Ibid.

58. Gauthier (1984). 
59. Le Guen $(1995,76)$.

60. Le Guen $(1995,81)$.

61. Hesberg $(2009,297)$.

62. Le Guen $(1995,80)$; Hesberg $(2015,103-108)$.

63. SEG 29, 1089, 1.7-8. Le théâtre de Théangéla n'a pas été découvert. Minniôn est vraisembablement l'un des amis d'Antiochos III : cf. Savalli-Lestrade (1998, $\mathrm{n}^{\circ} 34$ ).

64. Il existe toutefois des théâtres en dehors des centres civiques, dans des sanctuaires. Un théâtre est construit à proximité du sanctuaire d'Artémis à Amyzon : cf. Robert \& Robert (1983, 84-86). En Chersonèse de Carie, le sanctuaire d'Hémithéa reçoit un théâtre dès le début du III ${ }^{\mathrm{e}}$ siècle. Il accueille des représentations théâtrales et les réunions du koinon des Chersonésiotes : cf. Held (2013, 95-96) et Held \& Wilkening-Aumann $(2015,84)$.

65. Spanu $(2007,73)$ à propos des régions d'Anatolie centrale et méridionale.

66. Nysa et Aphrodisias n'ont pas de théâtre avant la deuxième moitié du $\mathrm{I}^{\text {er }}$ siècle: cf. Kadioğlu (2006) et Chaisemartin \& Theodorescu (2017). Seuls les plus petits centres urbains paraissent dépourvus de théâtre, comme Pidasa, Kodapè et Olymos.

67. En l'état actuel des recherches, et compte tenu de la présence possible d'édifices en matériaux périssables énoncée plus haut.

68. Isager $\left(2004, \mathrm{n}^{\circ} 1\right)$; Migeotte $\left(1984, \mathrm{n}^{\circ} 102\right)$. Sur l'architecture des gymnases, voir Delorme (1960) et Hoff (2009).

69. Gauthier (1995). Sur l'éphébie, voir Chankowski (2004) et Chankowski (2011).

70. Les gymnases sont abrégés « $G$ », les palestres « $P$ », les amphithéâtres « $A$ » et les stades « $S$ ». «HL » signifie époque hellénistique. Les noms de site entre parenthèses sont ceux pour lesquels un indice épigraphique laisse penser qu'un gymnase existe dans la cité. Les références en gras correspondent à la construction de l'édifice ou à des travaux.

71. Mert (2008, 20 et 157-166).

72. Dédicace de l'architrave de l'exèdre, photographiée et transcrite dans Mert (2008, 156-157).

73. IK, 34-Mylasa 137, 1. 21-23 ; Blümel (2004, 35-36).

74. Isager $\left(2004, \mathrm{n}^{\circ} 1\right)$.

75. Sur Bargasa, voir Debord \& Varinlioğlu (2010, $n^{\circ}$ 5) ; sur Mobôlla, voir HTC 64 et 65.

76. Gauthier (1995, 1, n. 6).

77. Sur les activités pratiquées dans les gymnases, voir Gauthier \& Hatzopoulos (1993).

78. Sur l'éphébie, voir Chankowski (2011). Sur les néoi, voir Dreyer (2004) et Bremen (2013).

79. Sur l'importance de ces pièces, voir Hesberg $(1995,19)$.

80. Robert (1960a, 298, n. 3).

81. Sur le culte d'Hermès et Héraclès, voir Delorme (1960, 339-340). Sur les cultes héroïques, voir Nilsson $(1955,64-67)$. Sur le culte de bienfaiteurs, voir Habicht $(1995,91)$. Sur le culte royal, voir Robert (1960b, 124-125) et Delorme (1960, 345).

82. Plusieurs exemples hors de Carie dans Schmitt-Pantel (1992, 370-371).

83. P. ex. à Halicarnasse ( $\mathrm{III}^{\mathrm{e}}$ siècle) : cf. Isager (2004, $\left.\mathrm{n}^{\circ} 2,1.27-34\right)$; à Alabanda : cf. Vitruve, De l'architecture, VII, 5, 6 .

84. Gauthier $(1995,1)$.

85. Voir infra n. 121.

86. Hesberg $(1995,13)$.

87. En Égypte, les Grecs sont «ceux du gymnase »: cf. Hesberg $(1995,16)$. À Jérusalem, l'entraînement au gymnase est le fait des Juifs hellénisés: cf. Lafon, Marc \& Sartre (2003, 185-186) ; Gehrke $(2004,417)$; Kobes $(2004,240-243)$.

88. Théâtre du bas à Cnide : cf. Bruns-Özgan (2013, 37-74); gymnase de Mylasa: cf. IK, 34Mylasa 21 ; bouleutérion d'Halicarnasse : cf. Isager (2004, $\left.\mathrm{n}^{\circ} 4 \mathrm{a}\right)$.

89. Sur Bargasa, voir Debord \& Varinlioğlu (2010, 145-152). Sur Cnide, voir Bankel (1997), Bankel (2009) et Ehrhardt (2009). Sur Kodapè, voir Varinlioğlu (2004, $\left.n^{\circ} 1=S E G 54,1164\right)$. Sur Iasos, voir 
Masturzo (1995). Sur Héraclée du Latmos, voir Peschlow-Bindokat $(1996,37)$ et PeschlowBindokat $(2005,120)$. Sur Mylasa, voir IK, 34-Mylasa 501.

90. Rostovtseff ([1941] 1989, 450); Bresson \& Descat $(2001,13)$.

91. Gauthier $(1985,72)$.

92. Sur l'évergétisme, voir Gauthier (1985) ; sur l'emprunt et les souscriptions publiques, voir Migeotte (1984) et Migeotte (1992) ; sur les financements royaux, voir Schmidt-Dounas (2000); sur les financements des constructions en général, voir Meier (2012).

93. IK, 34-Mylasa 21.

94. Isager $\left(2004, \mathrm{n}^{\circ} 1,1.20-21\right)$.

95. Cette souscription a probablement été initiée par le pouvoir dominant la communauté, Rhodes ou un souverain lagide ou séleucide : cf. Debord \& Varinlioğlu (2001, 102-103).

96. Debord \& Varinlioğlu (2001, 99-100).

97. $H T C \mathrm{n}^{\circ} 61$. L'objet de la souscription est inconnu.

98. Migeotte $(1984,362)$.

99. Meier $(2012,177)$.

100. Isager (2004, $\left.\mathrm{n}^{\circ} 4 \mathrm{a}, 1.3\right)$.

101. Le reste de la dépense a pu être assumé par le roi Ptolémée II ou III auquel le bâtiment est dédié, et par la cité, sur ses fonds propres.

102. Sur ce fonds, voir Migeotte (2006, 90-91).

103. Prytanée à Bargylia : cf. IK, 28.2-Iasos 612 ; agoranomion à Iasos : cf. IK, 28.1-Iasos 22.

104. Iasos : cf. IK, 28.1-Iasos 164 ; Maddoli (2007, $\left.\mathrm{n}^{\circ} 27.1\right)$; IK, 28.1-Iasos 179 ; LBW, 276 ; IK, 28.1Iasos 183 ; IK, 28.1-Iasos 182 ; IK, 28.2-Iasos 249. Stratonicée de Carie, proskènion : cf. Mert (2008, PS. AF. 2) ; IK, 22.1-Stratonikeia 1013.

105. IK, 34-Mylasa 137.

106. $I K$, 34-Mylasa 110.

107. IK, 34-Mylasa 110, 1. 9-11. Traduction Froehner (1865, $\mathrm{n}^{\circ}$ 98), modifiée.

108. Porte ouest (II siècle) : cf. Pedersen \& Isager $(2015,310)$.

109. Debord \& Varinlioğlu $\left(2018, n^{\circ} 21\right)$.

110. Debord \& Varinlioğlu $\left(2018, n^{\circ} 22\right)$.

111. Bresson (1991, $\mathrm{n}^{\circ} 38$ ), entre 320 et 281 . L'identification de l'édifice qualifié de naos est incertaine. Il pourrait s'agir d'un temple plus ancien que le grand temple visible aujourd'hui : cf. Debord (1982, 41-45). Un autre individu a également financé des colonnes du temple d'Hémithéa : cf. Held $(2013,96)$.

112. Ces actions sont souvent en faveur de gymnases: Philippe Arrhidée finance une palestre et un gymnase à Mylasa ca 317 (IK, 34-Mylasa 21). À Halicarnasse, Ptolémée II ou III pourrait être impliqué dans la restauration du gymnase au III ${ }^{\mathrm{e}}$ siècle: cf. Isager $\left(2004, \mathrm{n}^{\circ} 1\right)$. Antiochos III finance aussi les réparations d'une canalisation à Héraclée du Latmos au $\mathrm{II}^{\mathrm{e}}$ siècle: cf. Wörrle (1988).

113. Lettre de la reine Laodice aux Iasiens : cf. IK, 28.1-Iasos 4. Sur le séisme de 199-198: cf. Trogue Pompée dans Justin, Histoire Philippique, XXX, 4, 1-3 ; Guidoboni (1994, nº 41). En 213, Antiochos III contribue aussi à l'épanorthôsis de la ville de Sardes suite à son siège de la ville : cf. SEG 39, 1295, 1. 1 ; Gauthier $(1989,18)$.

114. Sur le théâtre : cf. IK, 28.2-Iasos 249 ; IK, 28.1-Iasos 164 ; Maddoli (2007, n² 27.1) ; IK, 28.1Iasos 179; LBW, 276; IK, 28.1-Iasos 183; IK, 28.1-Iasos 182; Crowther $(1990,150)$. Sur le bouleutérion et l'archeion : cf. IK, 28.2-Iasos 252.

115. Delrieux $(2001,138)$.

116. À Éphèse il est clair que Lysimaque n'a pas eu le temps de bâtir autre chose que les remparts et que ce sont les Séleucides qui ont entamé les premiers aménagements intra-muros : cf. Larguinat-Turbatte (2014). 
117. Wörrle (2003). Sur le site d'Héraclée du Latmos, voir Peschlow-Bindokat (1996) et PeschlowBindokat (2005).

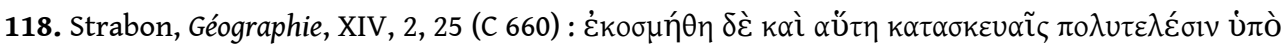
$\tau \tilde{\omega} v \beta \alpha \sigma \iota \lambda \varepsilon^{\prime} \omega v$. Cf. Robert (1937, 193, n. 1, 523, 534-535).

119. Debord $(2001,157)$.

120. Kenzler $(1999,303)$.

121. Sur la Carie et la Lycie, voir Prost $(2007,107)$, Piras $(2010,232)$ et Prost $(2013,186)$.

122. Ainsi en Pisidie-Pamphylie ou en Lycie où les théâtres et les bouleutéria sont rares à l'époque hellénistique : cf. Kolb (2013, 122-124) et Rivalland (2014).

123. Le lien entre les transformations sociales et l'aménagement urbain est mis en évidence par Pont (2010) au sujet de l'évergétisme. Sur la corrélation entre l'évolution du paysage urbain et les relations d'Aphrodisias avec Rome, voir Reynolds (1982) et Ratté (2002). Au sujet du théâtre, voir Chaisemartin \& Theodorescu (2017).

\section{RÉSUMÉS}

Dans la Carie hellénistique, la constitution d'un paysage urbain de type grec paraît étroitement liée au phénomène politique et culturel de l'adoption du modèle de la cité grecque. L'une des raisons de la construction d'édifices publics, en particulier ceux qui abritent les différentes institutions politiques et administratives de la cité, est qu'il existe une intense vie civique tout au long de l'époque hellénistique. De même, les communautés traduisent dans la pierre l'importance des pratiques culturelles qui prennent place dans les théâtres et dans les gymnases. En ce qui concerne les modalités de financement, les cités cariennes ont mis en œuvre des pratiques financières grecques qui ont favorisé la constitution progressive d'un paysage urbain caractéristique d'une cité grecque.

In Hellenistic Caria, the constitution of a cityscape of a Greek type is related to the political and cultural phenomenon of adoption of the Greek city-state model. One of the reasons why people built public buildings, especially those dedicated to political and administrative institutions, is that an intense civic life exists throughout the Hellenistic period. In the same way, communities express on stones the importance of cultural habits taking place in theatres and gymnasia. Concerning financing methods, Carian cities used Greek financial practices that helped the progressive constitution of the typical cityscape of a Greek city.

\section{INDEX}

Keywords : Caria, cityscape, Hellenistic period, theatre, gymnasium, city-state

Mots-clés : Carie, paysage urbain, époque hellénistique, théâtre, gymnase, cité 
AUTEUR

\section{GABRIÈLE LARGUINAT-TURBATTE}

Membre associée à l'IRAA (Aix-Marseille Université)

et Ausonius (Université Bordeaux Montaigne)

g.larguinatturbatte@gmail.com 